\title{
A STOCHASTIC MULTICLOUD MODEL FOR TROPICAL CONVECTION*
}

\author{
BOUALEM KHOUIDER ${ }^{\dagger}$, JOSEPH BIELLO ${ }^{\ddagger}$, AND ANDREW J. MAJDA ${ }^{\S}$ \\ Dedicated to the sixtieth birthday of Professor Andrew Majda
}

\begin{abstract}
A stochastic model for representing the missing variability in global climate models due to unresolved features of organized tropical convection is presented here. We use a Markov chain lattice model to represent small scale convective elements which interact with each other and with the large scale environmental variables through convective available potential energy (CAPE) and middle troposphere dryness. Each lattice site is either occupied by a cloud of a certain type (congestus, deep or stratiform) or it is a clear sky site. The lattice sites are assumed to be independent from each other so that a coarse-grained stochastic birth-death system, which can be evolved with a very low computational overhead, is obtained for the cloud area fractions alone. The stochastic multicloud model is then coupled to a simple tropical climate model consisting of a system of ODEs, mimicking the dynamics over a single GCM grid box. Physical intuition and observations are employed here to constrain the design of the models. Numerical simulations showcasing some of the dynamical features of the coupled model are presented below.
\end{abstract}

Key words. Stochastic modeling, Markov chains, tropical convection, clouds, convective parametrization, climate modeling, CAPE, moisture.

AMS subject classifications. 65C05, 65C20, 65C40, 65L05, 86A10, 76M35, 76R99.

\section{Introduction}

Stochastic methods and stochastic modelling are becoming increasingly important for both numerical simulations and theoretical studies in science and engineering. While Langevin-like stochastic differential equations constitute the benchmark for theoretical studies of slowly varying processes that interact with other processes evolving on much faster scales, which are often treated as a background noise, Monte Carlo simulations aim to track the detailed stochastic evolution of the microscopic processes, using physical first principles. Since the pioneering works of Gillespie (e.g. $[5,6])$ with his famous exact stochastic algorithms and the recent advancement in computational capabilities, Monte Carlo simulations are becoming more and more popular (e.g. [17]).

Although stochastic models have been around for decades, their use in large scale climate simulations is very recent. They are used essentially to account for the missing variability in general circulation models (GCMs) due to small-scale unresolved convective processes. Buizza et al. [1] used a stochastic backscattering model to represent the model uncertainties in a GCM and Lin and Neelin [45, 46] used a stochastic parametrization to randomize the way in which deep convection responds to large fluctuations via a prescribed probability distribution function for the convective time scale. Nonetheless, Majda and Khouider [35] are the first to propose a stochastic model for convective inhibition allowing both internal interactions between convective elements and between convective elements and the large scale/resolved variables.

\footnotetext{
*Received: October 8, 2008; accepted (in revised version): January 5, 2009.

†Department of Mathematics and Statistics, University of Victoria, PO BOX 3045 STN CSC, B.C. Victoria, Canada V8W 3P4, (khouider@uvic.ca).

${ }^{\ddagger}$ Department of Mathematics, University of California, Davis, One Shields Ave. Davis, CA 95616, (biello@math.ucdavis.edu).

$\S$ Centre for Atmosphere Ocean Science and Courant Institute of Mathematical Sciences, New York University, 251 Mercer Street, Room 902, New York, NY 10012, (jonjon@cims.nyu.edu).
} 
Their model is based on an Ising-type spin-flip model used earlier to model phase transitions in material sciences $[16,15,8]$. The stochastic dynamics are then coarse grained to derive a hierarchy of birth-death stochastic models, which are intermediate between the microscopic spin-flip dynamics and the mean field equation $[18,19]$ and thus very inexpensive to run on a computer. Such a coarse-grained birth-death process is used successfully by Khouider et al. [25] to couple a fully stochastic model for convective inhibition to a toy GCM. Further analysis of such small scale/stochastic models coupled to a large scale dynamical system addressing the important issues of phase transition, meta-stability and intermittency can be found in the series of papers by Katsoulakis et al. [11, 12, 13, 14]. In the same vein, Crommelin and Vanden Eijden [2], used detailed numerical simulation data to infer the conditional probability laws for a Markov process representing the subgrid scale modes for the Lorenz system.

Here, we propose to develop such intermediate stochastic models to represent cloud elements in a parametrization for organized tropical convection consisting of three cloud types of different height and vertical extent [20, 23, 24]. The cloud elements interact among themselves and with the environment based on some intuitive assumptions consistent with observations. The rest of the paper is organized as follows. An introductory discussion on moist convection and organized tropical convective systems is given in section 2 while the multicloud stochastic model is introduced in section 3. The computational grid box is divided into a rectangular lattice and convective elements evolve at each lattice site according to a Markov process whose states determine whether we have clear sky or a cloud of a certain type. It is shown in section 3 that the resulting set of Markov chains have a stationary-limiting distribution depending solely on the large scale/background state. In section 4, we derive an intermediate stochastic model for the evolution of cloud area fractions without having to evolve the microscopic site dynamics $[18,19,25]$ and deduce the mean-field equations. In section 5 , the multicloud stochastic model is coupled to a large scale tropical climate model which has a crude vertical resolution reduced to the two first baroclinic modes and a constant horizontal profile, mimicking the dynamics over one single GCM grid box - a one column model [20]. Some discussion and concluding remarks are given in section 6 .

\section{Organized convection and tropical convective systems}

Recent satellite observations reveal that convection in the tropics is organized into a hierarchy of space and time scales ranging from the individual convective cells (clouds) of one to 10 kilometres and a few hours, to mesoscale cloud clusters (also known as mesoscale convective systems) of a few hundreds of kilometres and one to two days, to super-clusters of a few thousands of kilometres and five to 10 days, to their planetary/intraseasonal scale envelopes known as the Madden-Julian oscillation $[30,44,49,50,7,31,3,9,53,10,26,40]$. The physical and dynamical features of organized convection and the associated interactions across scales are not all well understood. Furthermore, despite the recent progress in computing resources, the current general circulation models (GCMs) poorly represent these organized multiscale convective systems, partly due to the inadequate representation of unresolved features of organized convection by the cumulus parametrizations utilized in the GCMs $[47,42,43,41]$.

Nonetheless, it is has been recently recognized that tropical convective systems are qualitatively self-similar in terms of their cloud morphology and flow structure $[40,26,38,34,27]$. As noted by Mapes et al [40] and others, the heating field of tropical convective systems, for instance, from the mesoscale convective systems and 
squall lines $[55,56]$ to synoptic scale convectively coupled waves $[50,48]$ to the planetary/intraseasonal scale Madden-Julian oscillation [10, 26] involves three cloud types. Congestus cloud decks with a vertical extent which does not exceed the freezing level, at about 5 to $6 \mathrm{~km}$, in front, are followed by deep convective towers extending to the top of the troposphere, which in turn are lagged by stratiform anvils in their dissipation phase. Congestus clouds heat the lower troposphere due to condensational heating and induce upper troposphere cooling because of detrainment at their tops and thus serve essentially to precondition and moisten the middle troposphere. Deep convective towers dominate the core of the storm and are believed to be responsible for most of the tropical rainfall and provide the bulk heating for the whole tropospheric column. Stratiform anvil clouds, in the wake of the wave, heat the upper troposphere and cool the lower troposphere due to the evaporation of stratiform rain. A cartoon of the three cloud types is sketched in figure 2.1. While the transition

\section{Top of the troposphere}

(s)

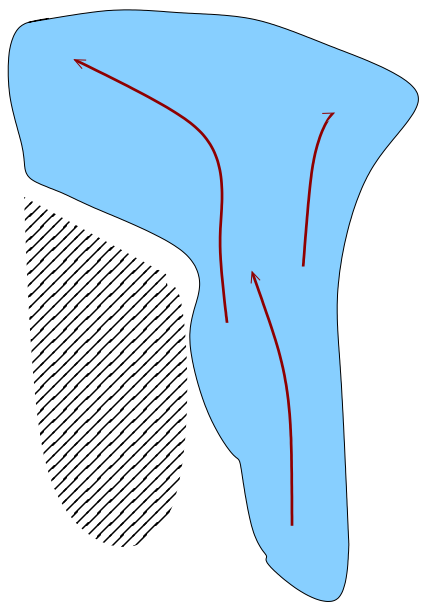

(d)

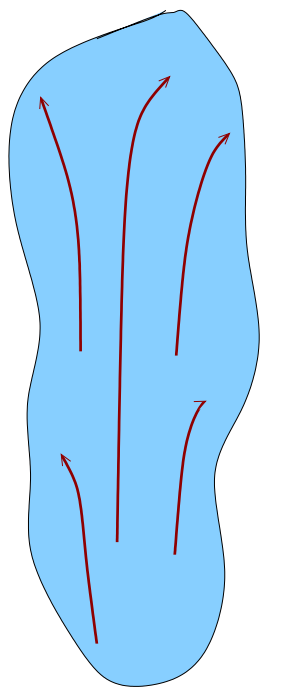

(c)

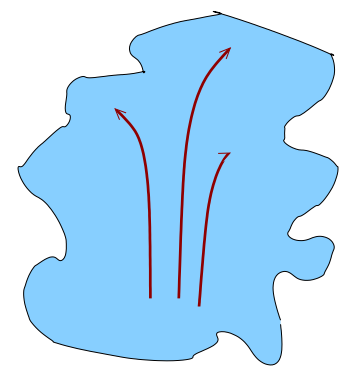

Top of the boundary layer

FiG. 2.1. A cartoon of the three cloud types showing congestus (c), deep convective (d), and a decaying deep convective tower with a lagging large stratiform anvil ( $s$ ), with stratiform rain falling into a dry region below it where it eventually evaporates and cools the environment (hatched area). The arrows indicate convective motion within the cloud.

from deep to stratiform is easily interpreted as the passing of the cloud from a liquid phase to an ice phase within the upper troposphere, the delay in the formation of deep convective towers is less obvious. A plausible explanation is given here in terms of the buoyancy of a rising convective parcel evolving in an environment whose moisture content is constantly changing, from dry to moist and vice-versa. Two different scenarios are sketched in figure 2.2: that of a dry environment (left) and that of a moist environment (right). Unlike dry convection where the fluid becomes unstable 
when the Rayleigh number exceeds its critical value, moist convection is subject to what is known as "conditional instability". Atmospheric air is stratified; it is stable to infinitesimal displacements because the displaced parcel cools down/warms up by expansion/compression and finds itself heavier/lighter than the surrounding air. But when a moist parcel is raised beyond some threshold height, the lifted condensational level, it becomes saturated and starts to condense liquid water, which is accompanied by latent heat release that compensates for the cooling due to pressure drop and the parcel becomes positively buoyant [4]. In the ideal situation, when the rising parcel doesn't exchange mass and/or heat with the environment its equivalent potential temperature, $\theta_{e p}$, is conserved (at least to a first order approximation). It thus follows the vertical dashed-straight line shown in figure 2.2, known as the moist adiabat, while the solid thick curve represents the saturation equivalent potential temperature, $\theta_{e}^{*}$, of the environment. The rising parcel remains positively buoyant as far as $\theta_{e p}>\theta_{e}^{*}$. The vertical integral of buoyancy of a parcel following the moist adiabat is called convectively available potential energy (CAPE). In reality however, the convecting parcel mixes with the environmental air as it rises and thus loses some of its buoyancy due to entrainment of dry air and would hit the environmental curve typically right above the turning point, which happens to lie below the freezing level when the middle troposphere is dry (left panel in figure 2.2). Such a dry situation is often observed in the front of tropical convective systems; it gives rise to cumulus congestus clouds which then serve to moisten the middle troposphere, partly because they detrain and dissipate and partly because they trigger low-level convergence of moisture $[20,23]$ and reduce the conditional stability of the environment by "pushing" the turning point of the $\theta_{e}^{*}$-curve to the right, thus preconditioning the environment for upcoming convective parcels, which can then rise beyond the freezing-level and gain extra buoyancy due to freezing and generate deep convective towers (right panel in figure 2.2).

The progressive deepening of convection from shallow to congestus to deep to stratiform is believed to be responsible for the front to rear tilt seen in the wind, temperature, and moisture fields of all convective complexes, at all scales, starting from mesoscale clusters and squall lines, to super-clusters, and then to the planetary scale MJO [53, 26]. However, tropical convective systems have genuine multiscale and multiphysics dynamics in the sense that the larger scale features are not just a simple linear/rescaling transformation of the smaller scale features, unlike homogeneous turbulence, but they enjoy many dynamical differences as well [54, 26, 27]. For instance the fluid mechanics of the MJO are different from those of a Kelvin wave, etc. Moreover, because of the causality principle of the three cloud types, Mapes et al. [40] arrived at the interesting conclusion that it is impossible to reproduce the hierarchy of tropical convective systems, having all the same-self-similar cloud morphology, etc., by a simple progressive embedding of a certain number of smaller entities into the larger ones, starting with three cloud type individual cells, as the building-blocks. Instead they propose a stretched building-block hypothesis where, within a mesoscale cloud cluster, there are mostly congestus clouds in front, mostly deep clouds in the centre and mostly stratiform anvils behind, as opposed to just congestus, just deep, and just stratiform, respectively. Therefore, on average (statistically speaking) the mesoscale convective system is an envelope of a certain number of convective cells with the same tri-cloud morphology (see their figure 11) with congestus cloud decks in front, deep convective clouds in the middle, and stratiform anvils behind. The synoptic scale super-clusters and planetary scale envelopes, in turn, are such that 
their front is formed by cloud clusters with mostly congestus clouds, their center is formed by cloud clusters with mostly deep clouds, and their back has cloud clusters with mostly stratiform cloud decks, etc.

Here we propose a stochastic lattice model, where each individual site represents one convective element, organized within each large scale grid box in a fashion mimicking the stretched building block paradigm of Mapes et al. [40]. Each lattice site is occupied by either a congestus, deep, or stratiform cloud or none (a clear sky site) according to certain probability rules depending on the large scale environment.
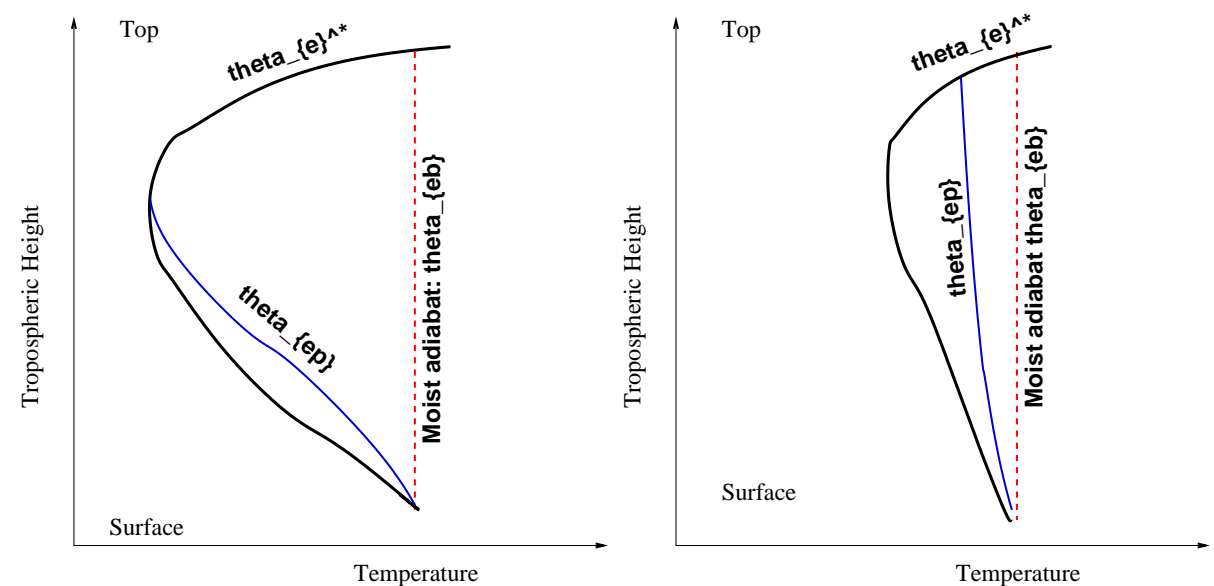

FIG. 2.2. Buoyancy of a rising parcel of moist air. The left panel sketches the case of a dry environmental sounding where the rising parcel, being mixed with the dry environmental air, loses its buoyancy before it reaches the freezing level and thus leads to the formation of congestus clouds. The right panel represents the case of the moist-preconditioned environment with a much smaller curvature allowing the rising parcel to reach the freezing level and thus gain extra-buoyancy, leading to the formation of deep penetrative towers. See text for details.

\section{The multicloud stochastic model}

We aim to represent the unresolved variability of organized tropical convection in a typical large scale-climate simulation with a mesh size of 100 to $200 \mathrm{~km}$. We consider a horizontal grid box for the tropical troposphere, above the planetary boundary layer, of rectangular shape, divided onto a lattice of $n \times n$ lattice points or sites. The parameter $n$ is a positive integer on the order 100 , so that the lattice sites are 1 to 2 kilometres apart, the typical scale for an individual cloud. Nonetheless, coarser lattices with mesh sizes of up to 10 to $15 \mathrm{~km}$ can be justified if one wants to take into account convective features such as large stratiform anvils and cold pools. A sensivity steady to the number of lattice sites is presented below. We assume that each lattice site is either occupied by a certain cloud type (congestus, deep, or stratiform) or it is clear sky, as shown in figure 2.3. A given site will switch from a given configuration to another according to some probability rules, which depend on the large scaleresolved variables. We thus construct a stochastic process at each lattice site taking the discrete values from zero to three according to whether the site is clear sky or occupied by a congestus, a deep, or a stratiform cloud, respectively. Notice that we are implicitly assuming that stratiform clouds exist as single entities, separated from the deep convective towers they originated from, as we don't assume that there is always a deep convective cloud site that is next to each stratiform anvil, unlike the cartoon 


\begin{tabular}{|l|l|l|l|}
\hline 0 & 1 & 0 & 2 \\
\hline 2 & 3 & 2 & 0 \\
\hline 0 & 0 & 1 & 0 \\
\hline 1 & 1 & 0 & 3 \\
\hline
\end{tabular}

FIG. 2.3. Lattice cloud model. A given lattice site is either clear sky (0) or occupied by a congestus cloud (1), a deep convective cloud (2), or a stratiform anvil cloud (3).

in figure 2.1 where the stratiform anvil is shown to be attached to a decaying deep convective tower. The three cloud type microscopic multi-state model is presented next.

3.1. The three cloud type microscopic multistate lattice model. Let $X_{t}^{i}, i=1, \ldots, n \times n$ and $t$ is time, be a continuous time stochastic process (Markov chain) such that at time $t$ we have

$$
X_{t}^{i}=\left\{\begin{array}{l}
0 \text { if site } i \text { is clear sky } \\
1 \text { if site } i \text { is occupied by a congestus cloud } \\
2 \text { if site } i \text { is occupied by a deep convective cloud } \\
3 \text { if site } i \text { is occupied by a stratiform anvil. }
\end{array}\right.
$$

For convenience, we assume that the transition probabilities among the four states $0,1,2,3$, at any given lattice site $i, i=1, \ldots, n \times n$, satisfy the following general requirements.

$$
\begin{aligned}
& P_{l k}^{i} \equiv \operatorname{Prob}\left\{X_{t+\Delta t}^{i}=k / X_{t}^{i}=l\right\}=R_{l k}^{i} \Delta t+o(\Delta t), \\
& \text { for } l, k=0,1,2,3 \text {, and } l \neq k
\end{aligned}
$$

and

$$
P_{l l}^{i} \equiv \operatorname{Prob}\left\{X_{t+\Delta t}^{i}=l / X_{t}^{i}=l\right\}=1-\sum_{k=0, k \neq l}^{3} P_{l k}^{i},
$$

where $\Delta t>0$ is a small time increment and the $R_{l k}^{i}$ 's are prescribed transition rates which for simplicity are assumed to depend solely on the large-scale/resolved variables according to the following intuitive interaction rules between the different cloud types and the environment.

1. A clear site turns into a congestus site with high probability if CAPE is positive and the middle troposphere is dry. 
2. A congestus or clear sky site turns into a deep convective site with high probability if CAPE is positive and the middle troposphere is moist.

3. A deep convective site turns into a stratiform site with high probability with a prescribed conversion rate, which may or may not depend on the state of the environment.

4. A cloudy site turns back to clear sky with a certain probability according to a prescribed decay time scale for each cloud type.

5 . It is very unlikely, during the short period of time $\Delta t$, for a clear sky or a congestus site to turn into a stratiform site, for a deep convective or stratiform site to turn into a congestus site, or for a stratiform site to turn into a deep convective site.

Notice that the assumption that the transition rates depend only on the large scale variables amounts to ignoring interactions between the lattice sites all together and it implies that the stochastic processes associated with the different sites are identical. Therefore, unless otherwise stated, in the remaining of the paper, we drop the superscript $i$ and consider only the generic process $X_{t}$ with the transition probabilities $P_{l k}$ and transition rates $R_{l k}$.

It follows immediately from Assumption 5 that

$$
R_{03}=R_{13}=R_{21}=R_{31}=R_{32}=0 .
$$

To a first order approximation the stochastic matrix for the discrete generic multistate Markov chain $X_{0}, X_{\Delta t}, X_{2 \Delta t}, \ldots$, for sufficiently small $\Delta t$, is given by

$$
M=\left[\begin{array}{cccc}
1-P_{01}-P_{02} & P_{01} & P_{02} & 0 \\
P_{10} & 1-P_{10}-P_{12} & P_{12} & 0 \\
P_{20} & 0 & 1-P_{20}-P_{23} & P_{23} \\
P_{30} & 0 & 0 & 1-P_{30}
\end{array}\right]
$$

where $P_{l k}=R_{l k} \Delta t+o(\Delta t)$. For a fixed large-scale state, the transition rates are constants, independent of time. It follows immediately that $X_{k \Delta t}, k=0,1,2, \ldots$, is a stationary Markov chain. Notice that the zeros in the matrix $M$ in (3.5) approximate the most improbable transitions according to (3.4).

Among all the physical quantities used to describe the state of the atmosphere, in a given large scale numerical model, two are considered to be important for both triggering and maintaining tropical convection, i.e, for the formation and decay of the three cloud types (congestus, deep, and stratiform). These quantities are the convective available potential energy (CAPE) and the relative moisture content, i.e, moistness or rather dryness of the middle of the troposphere. In practice both CAPE and the atmospheric dryness are well defined functions of the large scale moist thermodynamic variables. We consider these below in section 4, where we couple the stochastic model to a deterministic one-column model.

For the time being we assume that both CAPE and dryness are two external parameters represented by the letters $C$ and $D$, respectively, varying roughly between 0 and 2 .

Let

$$
\Gamma(x) \equiv\left\{\begin{array}{cc}
1-e^{-x} & \text { if } x>0 \\
0 & \text { Otherwise. }
\end{array}\right.
$$




\begin{tabular}{|c|c|c|c|}
\hline Time & description & Case 1 & Case 2 \\
\hline$\tau_{01}$ & formation of congestus & 1 hour & 3 hours \\
$\tau_{10}$ & decay of congestus & 5 hours & 2 hours \\
$\tau_{12}$ & conversion of congestus to deep & 1 hour & 2 hours \\
$\tau_{02}$ & formation of deep & 2 hours & 5 hours \\
$\tau_{23}$ & conversion of deep to stratiform & 3 hours & 0.5 hour \\
$\tau_{20}$ & decay of deep & 5 hours & 5 hours \\
$\tau_{30}$ & decay of stratiform & 5 hours & 24 hours \\
\hline
\end{tabular}

TABLE 3.1. Example of prescribed values of the time scale of formation or decay of each cloud type or of conversion of one cloud type to another.

Then according to the assumptions 1,2,3,4 given above, we let

$$
\begin{aligned}
& R_{01}=\frac{1}{\tau_{01}} \Gamma(C) \Gamma(D), \\
& R_{02}=\frac{1}{\tau_{02}} \Gamma(C)(1-\Gamma(D)), \\
& R_{10}=\frac{1}{\tau_{10}} \Gamma(D), \\
& R_{12}=\frac{1}{\tau_{12}} \Gamma(C)(1-\Gamma(D)), \\
& R_{20}=\frac{1}{\tau_{20}}(1-\Gamma(C)), \\
& R_{23}=1 / \tau_{23}, \quad R_{30}=1 / \tau_{30} .
\end{aligned}
$$

Note for instance that $R_{01}$ is zero when $C \leq 0$ or $D \leq 0$ and approaches $\tau_{01}^{-1}$ when $C$ and $D$ are sufficiently large and positive, consistent with Assumption 1 above. Here the $\tau_{l k}$ 's are prescribed time scales of formation or decay of the corresponding cloud type or of conversion of cloud type $l$ to cloud type $k$. There is no obvious way to chose their values. Based on physical intuition gained from observations, numerical simulations, and theory of tropical convection (see for e.g. [10, 39, 20, 23] and references therein), the rule of thumb is that the cloud life time is of the order of hours, that the rate of cloud formation is much faster than that of their decay, and that stratiform clouds should decay much more slowly than either congestus or deep. Here we consider the two effectively extreme cases of cloud interaction time scales, depicted in Table 3.1, to highlight some interesting features of the stochastic multicloud model parametrization.

In (3.6), we assumed for simplicity that the stratiform generation and decay rates, $R_{23}$ and $R_{30}$, are both independent of the large scale parameters $C, D$. However, there is no physical reason why this should be the case and obviously, the results would be sensitive to such dependence. To illustrate this point we also consider, in addition to (3.6), an example where $R_{23}$ increases slowly with CAPE, using the timescales associated with Case 2 of Table 3.1

$$
R_{23}=\frac{1}{\tau_{23}} \Gamma(\sqrt{C}) .
$$

3.2. The stationary distribution, cloud area fractions, and the equilibrium statistics of the lattice model. The equilibrium distribution, $\mathcal{P}_{e}$, of the multistate Markov chain $X_{t}$ introduced above is given by the left eigenvector of the stochastic matrix $M$ associated with the eigenvalue one. It is given by 


$$
\mathcal{P}_{e}=\frac{1}{Z}\left(\begin{array}{c}
1 \\
\frac{R_{01}}{R_{10}+R_{12}} \\
\frac{1}{R_{20}+R_{23}}\left(R_{02}+\frac{R_{12} R_{01}}{R_{10}+R_{12}}\right) \\
\frac{R_{23}}{R_{30}} \frac{1}{R_{20}+R_{23}}\left(R_{02}+\frac{R_{12} R_{01}}{R_{10}+R_{12}}\right)
\end{array}\right)
$$

where $Z$ is a normalization constant, so that the entries of $\mathcal{P}_{e}$ sum to one.

Next, we define the area fractions $\sigma_{c}, \sigma_{d}, \sigma_{s}$ occupied by clouds of type congestus, deep, or stratiform at any given time $t$, as the number of lattice sites for which $X_{t}=1,2,3$, respectively, divided by the total number of sites $N=n \times n$ :

$$
\sigma_{c}=\frac{1}{N} \sum_{i=1}^{N} \mathbb{1}_{\left\{X_{t}^{i}=1\right\}}, \quad \sigma_{d}=\frac{1}{N} \sum_{i=1}^{N} \mathbb{1}_{\left\{X_{t}^{i}=2\right\}}, \quad \sigma_{s}=\frac{1}{N} \sum_{i=1}^{N} \mathbb{1}_{\left\{X_{t}^{i}=3\right\}}
$$

where

$$
\mathbb{1}_{\left\{X_{t}^{i}=k\right\}}=\left\{\begin{array}{lc}
1 & \text { if } X_{t}^{i}=k \\
0 & \text { otherwise. }
\end{array}\right.
$$

The clear sky area fraction is given by

$$
\sigma_{c s} \equiv 1-\sigma_{c}-\sigma_{d}-\sigma_{s}
$$

For all practical purposes the area fraction vector $\left(\sigma_{c s}, \sigma_{c}, \sigma_{d}, \sigma_{s}\right)$ can be interpreted as (and is equal to) the probability distribution of the generic stochastic process $X_{t}$ at time $t$. Therefore, the equilibrium distribution $\mathcal{P}_{e}$ in (3.8) yields the long time statistical equilibrium for the filling fractions $\sigma_{c}, \sigma_{d}, \sigma_{s}$.

A first test for the stochastic multicloud model is whether, on average, the equilibrium distribution should favour either congestus, deep, stratiform, or clear sky sites to form depending on the environmental conditions, according to the intuitive rules $1,2,3,4$. In figure 3.1, we plot on each corresponding panel the equilibrium clear sky, congestus, deep, and stratiform area fractions, as functions of CAPE, $C$, and dryness, $D$, for the $\tau_{l k}$ values in Table 3.1, Case 1 . We consider both the cases when (A) $R_{23}$ is independent on the large scale/external variables as given in (3.6) and (B) when $R_{23}$ is given by (3.7). As expected, both cases depict the general behaviour that for small CAPE values, independent of the dryness, we have clear sky dominated equilibrium and when both CAPE and dryness are large congestus dominates while deep convection and stratiform anvils dominate when CAPE is positive and the environment is moist. However when $R_{23}$ is also allowed to depend on CAPE (B) deep convective coverage becomes more important for moderate values of CAPE while stratifrom clouds dominate when CAPE is large. Although not shown here, an important factor, which somehow determines the average life time of a given cloud type, is the ratio between the generation and decay time scales. For instance when both the stratiform decay time, $\tau_{30}$, is increased from 5 hours to 10 hours and the time of conversion of deep to stratiform is decreased from 3 hours to 1.5 hours, the strength of stratiform cloudiness is increased considerably, especially for high values of CAPE, at the expense of the deep cloud coverage. Therefore, regardless of the large scale/external parameters $C, D$, the $\tau_{l k}$ 's provide a nice set of parameters to tune in order to set the desired cloud coverage configuration at equilibrium. The equilibrium cloud area 
fractions are of fundamental importance for the dynamics of large scale/deterministic convective parametrizations [33, 32]. Also, clearly the choice of the reference CAPE and dryness values used (below in section 5) for the renormalization of $C$ and $D$ are both crucial for the dynamics of the stochastic cloud fractions as they actually set the way in which the subgrid model responds to variations in CAPE and moistness in the middle of the troposphere.

We used a Monte Carlo method to simulate the sequence of Markov chains $X_{t}^{i}$, $i=1,2, \ldots N$, associated with each one of the lattice sites, employing an acceptancerejection algorithm $[5,6,28]$ where the times until each transition occurs are assumed to be independent exponential random variables. A maximum of two random numbers are thus generated at each iteration and for each lattice site, conditional on the states $0,1,2,3$. Recall that state 0 can change to state 1 or state 2 , state 1 can go to either 0 or 2 , and 2 can go to either 0 or 3 , while state 3 can go only to 0 . The first random number determines whether we make a change or not and the second random number determines if we go up or down, accordingly in the hierarchy of states. Only one random number is generated for state 3 , since only one change (3 to 0 ) is permitted.

As a test case, we let $C=0.25$ and $D=0.75$ : a relatively moist middle troposphere with a moderate but positive CAPE value. Starting with a random initial lattice configuration, we integrate the stochastic lattice model for about 100 hours, with $n=20$ and the typical time scales displayed in Table 3.1, Case 1. A snapshot (single realization at some fixed time) of the lattice state is shown in figure 3.2 (a) while the associated time series of the area fractions for each cloud type are shown in figure 3.2 (b), with the corresponding equilibrium values, from (3.8), are overlaid. Starting initially with a random lattice configuration, the cloud coverage fractions relax quickly to their corresponding equilibrium values and fluctuate around them with a significant variability of about $5 \%$ to $25 \%$ of the total area.

\section{Averaged birth-death stochastic model and the mean-field equa-} tions

Clearly, for a large number of sites of about $100 \times 100$, the full Monte Carlo simulation of evolving the $100 \times 100$ Markov chains all at once becomes impractical. However, for small enough $\Delta t$ one can argue that because the probability that only one site will undergo a change is much higher than the probability that more than one site undergoes a transition, for all practical purpose it is equivalent to testing whether one site at a time will undergo a transition or not; rather than testing them all at once (see [17] and references therein). Moreover, it is possible to derive the stochastic dynamics for the cloud coverages alone, which can be evolved without the detailed knowledge of the micro-state configuration, by using a coarse-graining technique $[18,19,25]$ that yields here a system of three birth-death-like processes, corresponding to the three cloud types.

Let $N=n \times n$ be the total number of lattice sites. Let $N_{c}^{t}$ be the number of congestus sites, $N_{d}^{t}$ the number of deep convective sites, and $N_{s}^{t}$ the number of stratiform sites, inside the lattice, at any given time $t \geq 0$. The number of clear sky sites is $N_{c s}^{t}=N-N_{c}^{t}-N_{d}^{t}-N_{s}^{t}$, by conservation of the total number of sites. Next, we compute the (transition) probabilities for the numbers (random variables) $N_{c}^{t}, N_{d}^{t}, N_{s}^{t}$ to go up or down during the small interval of time $(t, t+\Delta t]$.

We have

$$
\operatorname{Prob}\left\{N_{c}^{t+\Delta t} \geq k+1 / N_{c}^{t}=k\right\}=\sum_{i=1}^{N} \operatorname{Prob}\left\{X_{t}^{i}=0\right\} P_{01}^{i}+o(\Delta t),
$$



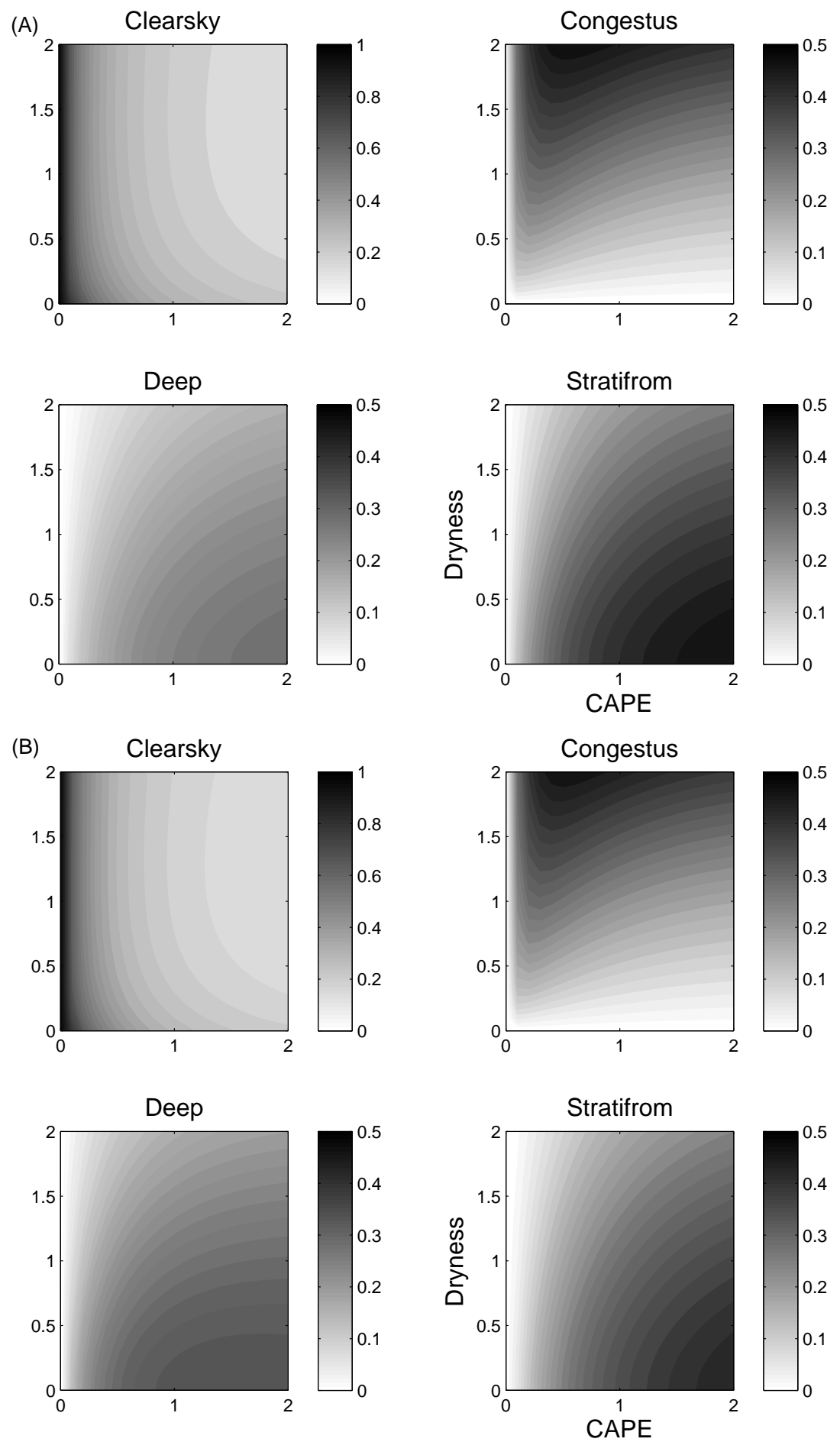

FIG. 3.1. Contour plots of the equilibrium area fractions as given by the stationary distribution in (3.8) in terms of CAPE and dryness parameters $C$ and D. Note the difference in color scales between the clear sky and cloud types filling fractions. (a) The transition rates are as (3.6) and the $\tau_{l k}$ 's are as in Table 3.1, Case 1, and (b) is same as (a) except for $R_{23}$ is given by (3.7). 

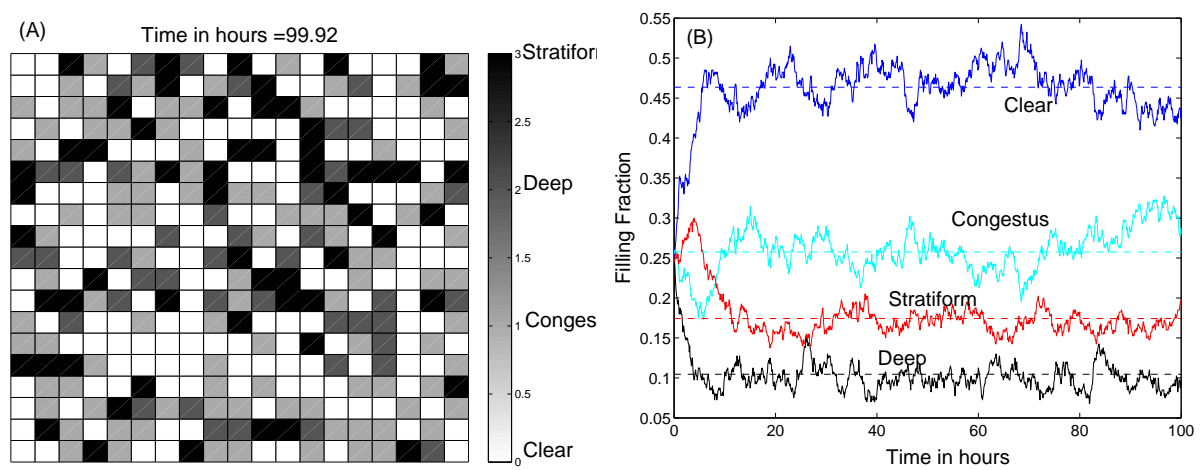

FIG. 3.2. An example of Monte Carlo simulation of stochastic multicloud model with $n=20, C=$ $0.25, D=0.75$, and the cloud time scales are as in Table 3.1, Case 1. (A) A snapshot picture of one typical lattice configuration and $(B)$ time series of the total coverages associated with each cloud type with the equilibrium values overlaid (dashed lines).

i.e, the probability that the number of congestus sites goes up by at least one is the sum of all the probabilities that a given clear sky site will turn into a congestus site. Given that all the sites are identical, i.e, the transition probability $P_{l k}^{i}$ is independent of $i$, if in addition we assume that initially the lattice configuration is uniform, then we have (see (3.9))

$$
\begin{aligned}
& \operatorname{Prob}\left\{X_{t}^{i}=1\right\}=\frac{N_{c}^{t}}{N}=\sigma_{c}^{t}, \quad \operatorname{Prob}\left\{X_{t}^{i}=2\right\}=\frac{N_{d}^{t}}{N}=\sigma_{d}^{t}, \\
& \operatorname{Prob}\left\{X_{t}^{i}=3\right\}=\frac{N_{s}^{t}}{N}=\sigma_{s}^{t}, \quad \operatorname{Prob}\left\{X_{t}^{i}=0\right\}=\frac{N_{c s}^{t}}{N}=\sigma_{c s}^{t}, \quad \forall i=1,2, \ldots, N .
\end{aligned}
$$

Therefore, using the fact that a single transition is most likely to occur when $\Delta t$ is small - allowing only one change at a time (which is equivalent to assuming that $N_{c}, N_{d}, N_{s}$ are combinations of Poisson processes), we arrive at

$$
\operatorname{Prob}\left\{N_{c}^{t+\Delta t}=k+1 / N_{c}^{t}=k\right\}=N_{c s} P_{01}+o(\Delta t)=N_{c s} R_{01} \Delta t+o(\Delta t) .
$$

Similarly, we have

$$
\begin{aligned}
\operatorname{Prob}\left\{N_{c}^{t+\Delta t}=k-1 / N_{c}^{t}=k\right\} & =\sum_{i=1}^{N} \operatorname{Prob}\left\{X_{t}^{i}=1\right\}\left(P_{10}^{i}+P_{12}^{i}\right)+o(\Delta t) \\
& =N_{c}\left(R_{10}+R_{12}\right) \Delta t+o(\Delta t), \\
\operatorname{Prob}\left\{N_{d}^{t+\Delta t}=k+1 / N_{d}^{t}=k\right\} & =\sum_{i=1}^{N} \operatorname{Prob}\left\{X_{t}^{i}=0\right\} P_{02}^{i}+\operatorname{Prob}\left\{X_{t}^{i}=1\right\} P_{12}^{i}+o(\Delta t) \\
& =\left(N_{c s} R_{02}+N_{c} R_{12}\right) \Delta t+o(\Delta t), \\
\operatorname{Prob}\left\{N_{d}^{t+\Delta t}=k-1 / N_{d}^{t}=k\right\} & =\sum_{i=1}^{N} \operatorname{Prob}\left\{X_{t}^{i}=2\right\}\left(P_{20}^{i}+P_{23}^{i}\right)+o(\Delta t) \\
& =N_{d}\left(R_{20}+R_{23}\right) \Delta t+o(\Delta t),
\end{aligned}
$$




$$
\begin{aligned}
\operatorname{Prob}\left\{N_{s}^{t+\Delta t}=k+1 / N_{s}^{t}=k\right\} & =\sum_{i=1}^{N} \operatorname{Prob}\left\{X_{t}^{i}=2\right\} P_{23}^{i}+o(\Delta t) \\
& =N_{d} R_{23} \Delta t+o(\Delta t), \\
\operatorname{Prob}\left\{N_{s}^{t+\Delta t}=k-1 / N_{s}^{t}=k\right\} & =\sum_{i=1}^{N} \operatorname{Prob}\left\{X_{t}^{i}=3\right\} P_{30}^{i}+o(\Delta t) \\
& =N_{s} R_{30} \Delta t+o(\Delta t) .
\end{aligned}
$$

The time sequences $N_{x}^{t}, x=c s, c, d, s$ are thus viewed as a coupled system of birthdeath Markov processes whose transition probabilities are given by (4.2) to (4.7), which can be easily evolved in time using Gillespie's exact algorithm [5, 6], while the cloud coverages are recovered according to (4.1), consistent with (3.9). In practice, we can also view this coupled birth-death system as a multistate/multivariable Markov chain undergoing one of the following seven transitions at a time: one congestus is formed from a clear sky, one deep is formed from a clear sky, one congestus is converted to deep, one deep is converted to stratiform, or one cloudy site turns to clear sky. The associated transition probabilities are given by the the original rates in (3.6) multiplied by the total number of sites that are subject to the given transition in a way which is consistent with the formulae (4.2) to (4.7). For example the rate of transition from clear sky to congestus is $N_{c s} R_{01}$ and the rate of convection of congestus to deep is $N_{c} R_{12}$, etc.

As one would expect, the dynamics of the area fractions obtained by evolving the full microscopic lattice-model, described in the previous section, through the detailed description of each one of the stochastic processes, $X_{t}^{i}$ are statistically equivalent to those obtained by evolving the coarse-grained birth-death processes described in this section. This is confirmed by numerical tests, which are not shown here. However, it is important to note that the computations are orders of magnitude cheaper in the latter case: Compare generating and testing seven random numbers versus $2 \times n \times n$ where $n$ is the total number of sites.

Normalizing by the total number of sites $N$ and dividing by $\Delta t$, the equations in (4.2) to (4.7) yield the mean field equations for the cloud coverages $\sigma_{c}, \sigma_{d}, \sigma_{s}$, given by the following third order system of ODEs:

$$
\begin{aligned}
& \dot{\sigma}_{c}=\left(1-\sigma_{c}-\sigma_{d}-\sigma_{s}\right) R_{01}-\sigma_{c}\left(R_{10}+R_{12}\right) \\
& \dot{\sigma}_{d}=\left(1-\sigma_{c}-\sigma_{d}-\sigma_{s}\right) R_{02}+\sigma_{c} R_{12}-\sigma_{d}\left(R_{20}+R_{23}\right) \\
& \dot{\sigma}_{s}=\sigma_{d} R_{23}-\sigma_{s} R_{30} .
\end{aligned}
$$

Note that the growth and decay rates of the mean field variables in (4.8) are given respectively by the birth and death rates in (4.2) to (4.7). This is a non-homogeneous linear system of ODEs with a unique equilibrium solution corresponding to the stationary distribution in (3.8), if all the transition rates in (3.6) are nonzero.

In figure 4.1, we plot the contours of the real and imaginary parts of the eigenvalues of the matrix (corresponding to the system) in (4.8) as functions of the parameters $C$ and $D$, using the time scales from Table 3.1, Case 1. As we see from figure 4.1, the equilibrium of the mean field equation (4.8) goes from an asymptotically stable node to a stable spiral as $C$ is increased. In other words this system bifurcates from an exponentially damped regime to an oscillatory damped regime as $C$ is increased away from zero: For large values of $C$ we distinguish one real negative eigenvalue and a pair of complex conjugate eigenvalues whose real part is negative while for small 
values of $C$, and only slightly depending on the values of $D$, we have three negative real eigenvalues. The imaginary part increases significantly with increasing values of $C$, especially for slightly moist conditions corresponding to $D$ between 0.2 and 0.3 . The damping strength is also sensitive to changes in $C$ and $D$. This behaviour of the mean field model may provide some insight into the behaviour of the stochastic system $[11,12,13,14]$. One could argue that an important non-dimensional number for the stochastic multicloud model is given by the ratio of the frequency to the damping rate, for the complex conjugate pair, plotted in Figure 4.2(E). In Figure 4.2, we display two time series of the area coverages obtained by evolving the stochastic model with $D=0.4$ and the two different values of $C=0.1$ and $C=1.5$. According to figure $4.2(\mathrm{E})$, the case $C=0.1$ has a frequency to damping ratio near zero (below 0.1 ) while in the second case this ratio is above 0.6. As anticipated, the two series are qualitatively different with the one corresponding to $C=1.5$ having sharper peaks and the one corresponding to $C=0.1$ has much longer excursions. Therefore, we conjecture here that a large frequency to damping ratio, in a complex conjugate pair for the mean field equations, would yield sharp and rapid oscillations for the associated stochastic system, while a small ratio would yield smoother oscillations with much longer excursions from equilibrium.
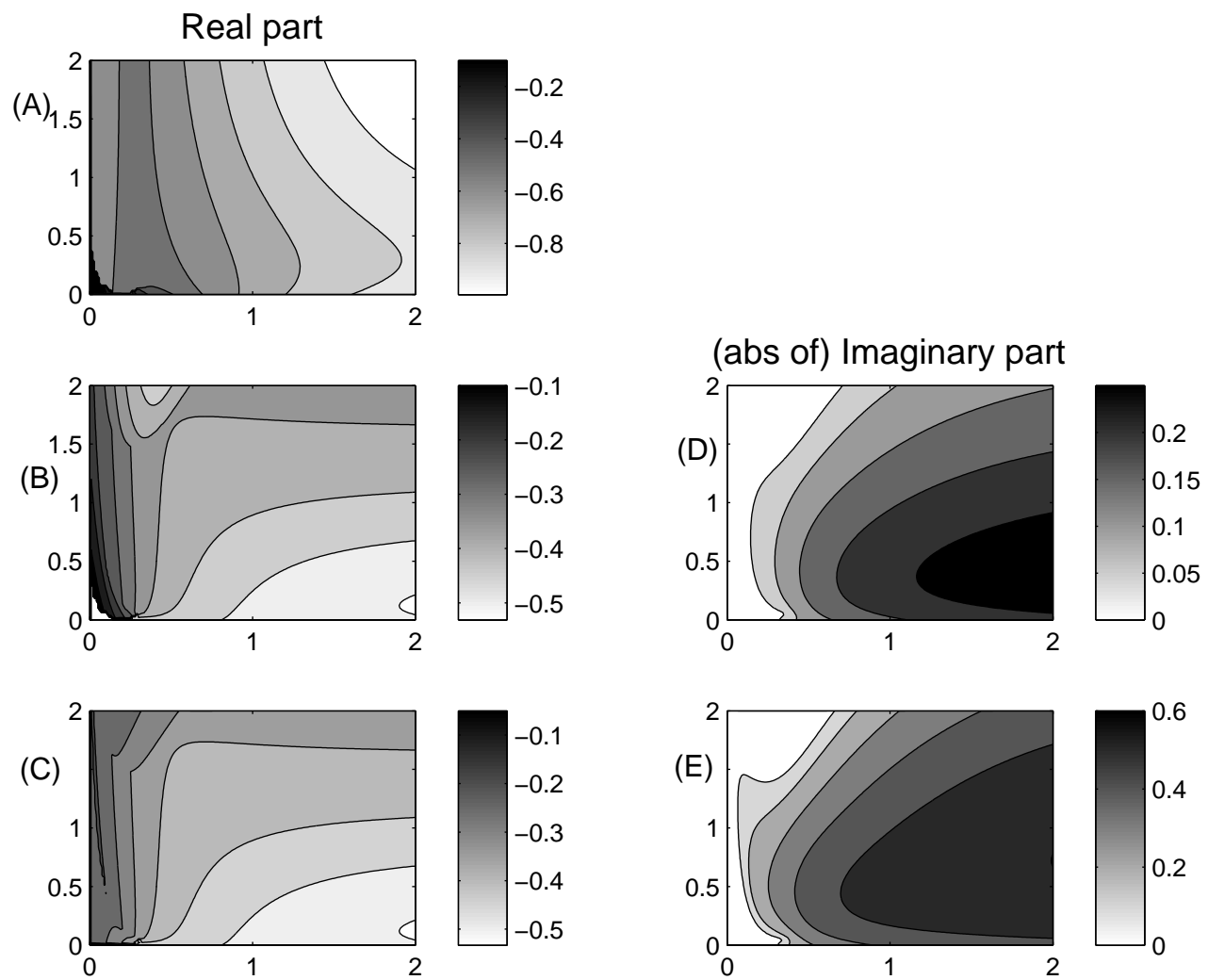

FIG. 4.1. Equilibrium eigenvalues of the mean field equations. Panels (A), (B), and (C) represents the contours of the real parts of the three eigenvalues, respectively, as CAPE $C$ (horizontal axis) and dryness $D$ (vertical axis) are varied from 0 to 2, Panel (D) shows the imaginary part of the complex conjugate pair, and Panel (E) displays the ratio of the frequency over the damping rate. 

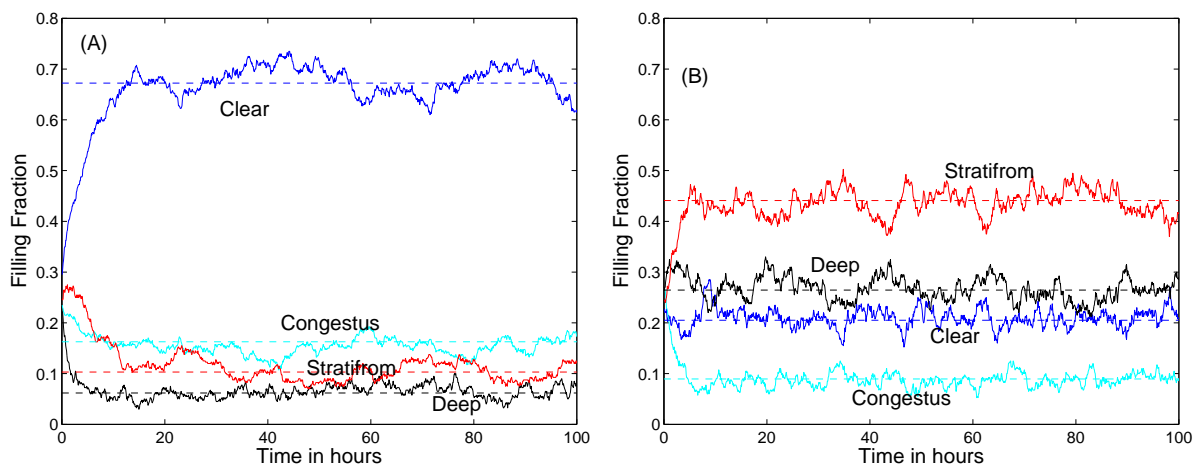

FIG. 4.2. Stochastic oscillations for both (a) when the frequency to damping ratio is small and (b) when it is large for the parameter values $D=0.4$ and $C=0.1$ and $C=1.5$, respectively, and the $\tau_{l k}$ 's are as in Table 3.1.

5. Coupling to a deterministic convective parametrization: case of a one-column model

As mentioned earlier, one of the main objectives of the multicloud stochastic model is to account for the unresolved variability due to cloud dynamics and cloudcloud interactions in a global numerical simulation of the atmospheric circulation. Typically, the general circulation models (GCMs) used by climate and weather forecasters solve the primitive equations on a large grid and all the unresolved physical processes are represented by a set of deterministic closures (in the form of differential and/or algebraic equations) known as parametrizations. In a series of papers, two of the authors have been developing and testing such parameterization models for organized tropical convection, with a crude vertical resolution reduced to the first two baroclinic mode/shallow water-like equations. This is the minimum possible vertical resolution which can account for a linear response to the heating fields induced by the three cloud types considered here: congestus, deep, and stratiform [20, 22, 23, 24]. As a further simplification, and in order to test temporal variability alone, in this paper we neglect the spatial dependence in the deterministic large scale convective parameterization equations and simply couple the stochastic multicloud-lattice model, described above, to the following simple ODE system [21, 36]:

$$
\begin{aligned}
\frac{\partial \theta_{1}}{\partial t} & =H_{d}-Q_{R, 1}^{0}-\frac{\theta_{1}^{\prime}}{\tau_{R}} \\
\frac{\partial \theta_{2}}{\partial t} & =H_{c}-H_{s}-Q_{R, 2}^{0}-\frac{\theta_{2}^{\prime}}{\tau_{R}} \\
\frac{\partial \theta_{e b}}{\partial t} & =\frac{1}{\tau_{e}}\left(\theta_{e b}^{*}-\theta_{e b}\right)-\frac{1}{h} D_{m} \\
\frac{\partial q}{\partial t} & =-P+\frac{1}{H_{T}} D_{m} .
\end{aligned}
$$

Here $\theta_{1}, \theta_{2}$ are the potential temperature components, associated with the first and second baroclinic modes, where the primed quantities represent a deviation from a (radiative-convective) equilibrium solution, so that the total potential temperature perturbation (in non dimensional units) is given by

$$
\theta^{\prime}=\theta_{1}^{\prime} \sin z+2 \theta_{2}^{\prime} \sin (2 z), 0 \leq z \leq \pi H_{T},
$$




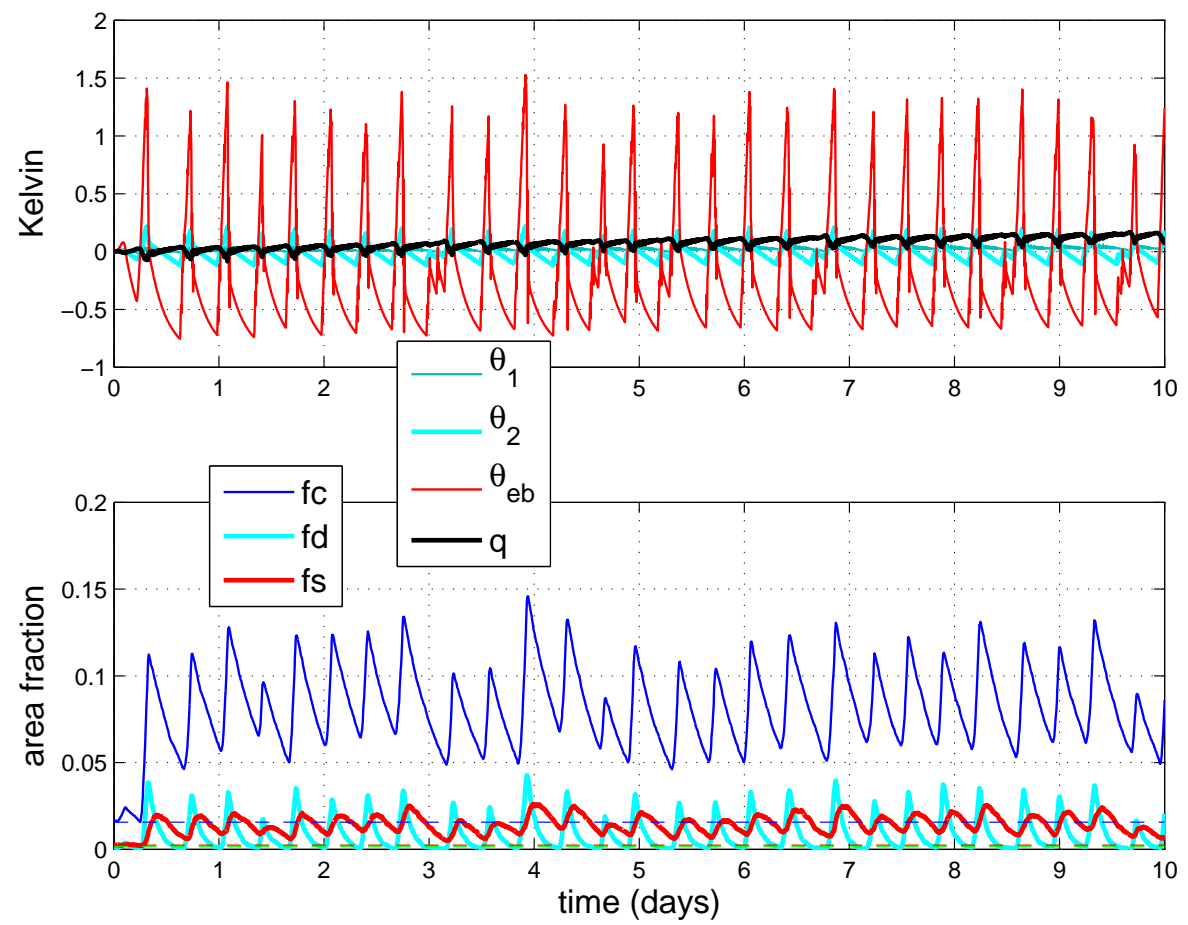

FIG. 5.1. Time series of the large-scale variables (top) and the (stochastic) cloud area fractions (bottom) for the parameter values corresponding to Case 1 in Table 5.2 with $\gamma_{2}^{\prime}=4$ and number of lattice sites is $100 \times 100$. The dashed lines on the bottom panel are the corresponding initial-RCE area fractions.

where $\theta_{e b}$ is the equivalent potential temperature in the boundary layer and $q$ is the vertically averaged water vapor content in the free troposphere [20,21], above the boundary layer ( $q$ is rescaled in units of temperature). The forcing terms $H_{d}, H_{c}, H_{s}$ are heating rates associated with deep convection, congestus, and stratiform clouds, respectively. $P$ and $D_{m}$ are, respectively, the precipitation rate and downdraft mass flux, exchanged between the free troposphere and boundary layer and $\frac{1}{\tau_{e}}\left(\theta_{e b}^{*}-\theta_{e b}\right)$ accounts for evaporation effects from the ocean surface with $\theta_{e b}^{*}$ being the saturation equivalent potential temperature in the boundary layer and $\tau_{e}$ the evaporation time scale. The terms $Q_{R, 1}^{0}$ and $Q_{R, 2}^{0}$ are fixed cooling rates due to long wave radiation while $\tau_{R}$ is a Newtonian cooling time scale. As in Khouider and Majda [20, 23], here

$$
P=\frac{2 \sqrt{2}}{\pi} H_{d}, \quad D_{m}=m_{0}\left(1+\mu\left(H_{s}-H_{c}\right)\right)^{+}\left(\theta_{e b}-\theta_{e m}\right)
$$

where $\mu>0$ is a tuning parameter, $m_{0}$ is a fixed constant, representing the downdraft mass flux scale, and $\theta_{e m}$ is the equivalent potential temperature in the middle of the troposphere where $\theta_{e m}^{\prime}=q^{\prime}+\frac{2 \sqrt{2}}{\pi}\left(\theta_{1}^{\prime}+\alpha_{2} \theta_{2}^{\prime}\right)$.

According to (5.2), the deep convective heating $H_{d}$ has a half sine profile in the vertical and thus serves to heat the entire troposphere while the congestus and stratiform heating rates $H_{c}, H_{s}$ have a full sine profile, thus they serve to heat and cool the lower troposphere and cool and heat the upper troposphere, respectively. Based 

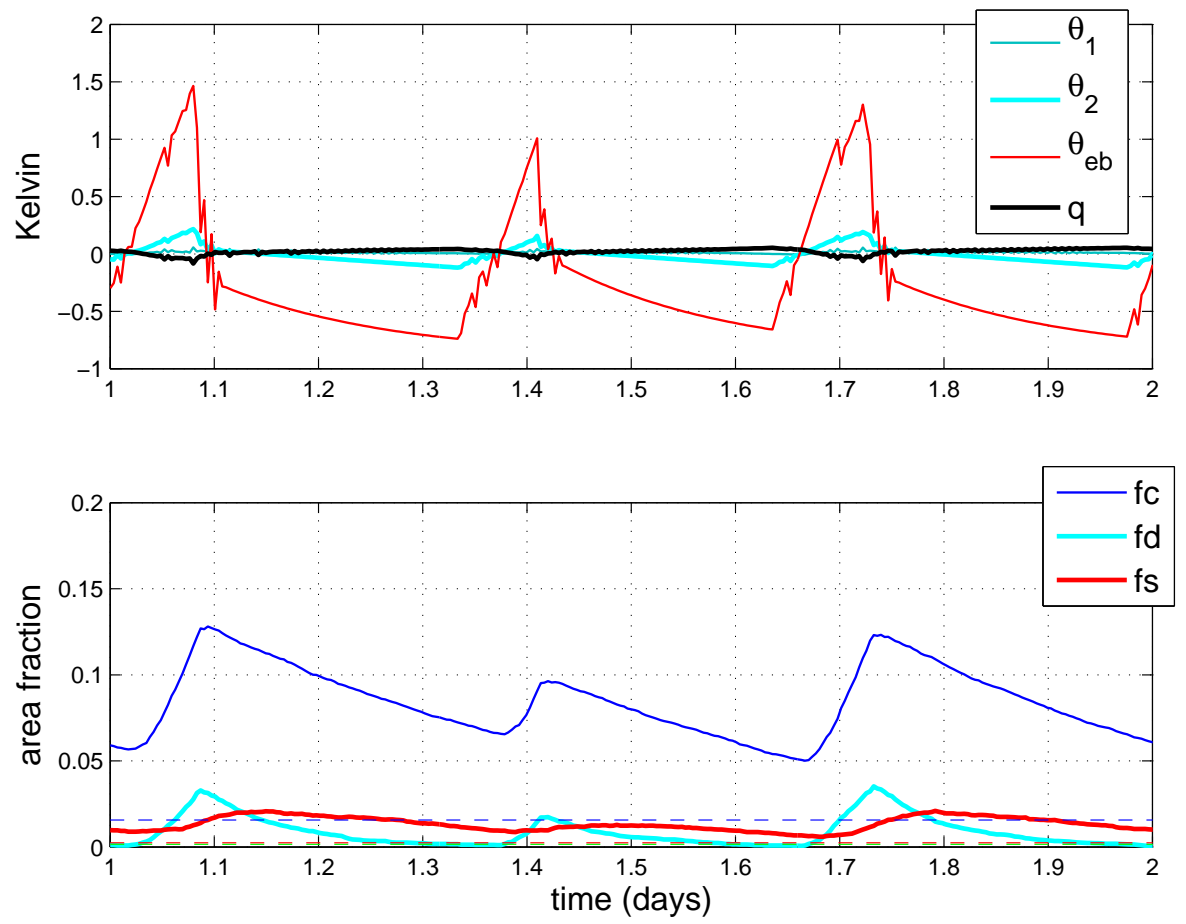

FIG. 5.2. Same as figure 5.1 except results are shown for the short period of one day.

on physical intuition, consistent with observations of tropical convective systems [29, 10, 53, 26] and detailed (cloud resolving) numerical simulations [52, 51], and earlier work [39, 33], Khouider and Majda [20, 21, 22, 23, 24] introduced deterministic closure formulas for each one of the heating rates which accounted for the causal dependence between the three cloud types depending on the large scale moisture and CAPE distributions. Especially, the progressive deepening of convection from shallow to deep is successfully captured by a moisture switch function, varying between zero and one according to the moistness and dryness of the middle troposphere. Here we circumvent this purely deterministic moisture function, although it is very plausible and technically simple, and assume that the strength of the heating rates due to the three cloud types is proportional to the area coverages $\sigma_{c}, \sigma_{d}, \sigma_{s}$ inferred directly from the stochastic lattice model presented in the previous sections. Thus, the following closure formulas are assumed here:

$$
\begin{aligned}
H_{d} & =\left(\sigma_{d} \bar{Q}+\frac{1}{\tau_{c}\left(\sigma_{d}\right)}\left(a_{1} \theta_{e b}^{\prime}+a_{2} q^{\prime}-a_{0}\left(\theta_{1}^{\prime}+\gamma_{2} \theta_{2}^{\prime}\right)\right)\right)^{+} \\
\tau_{c}\left(\sigma_{d}\right) & =\frac{\bar{\sigma}_{d}}{\sigma_{d}} \tau_{c}^{0} \\
H_{s} & =\sigma_{s} \frac{\bar{\alpha} \alpha_{s}}{H_{m}} \sqrt{\mathrm{CAPE}^{+}} \\
H_{c} & =\sigma_{c} \frac{\bar{\alpha} \alpha_{c}}{H_{m}} \sqrt{\mathrm{CAPE}_{l}^{+}},
\end{aligned}
$$




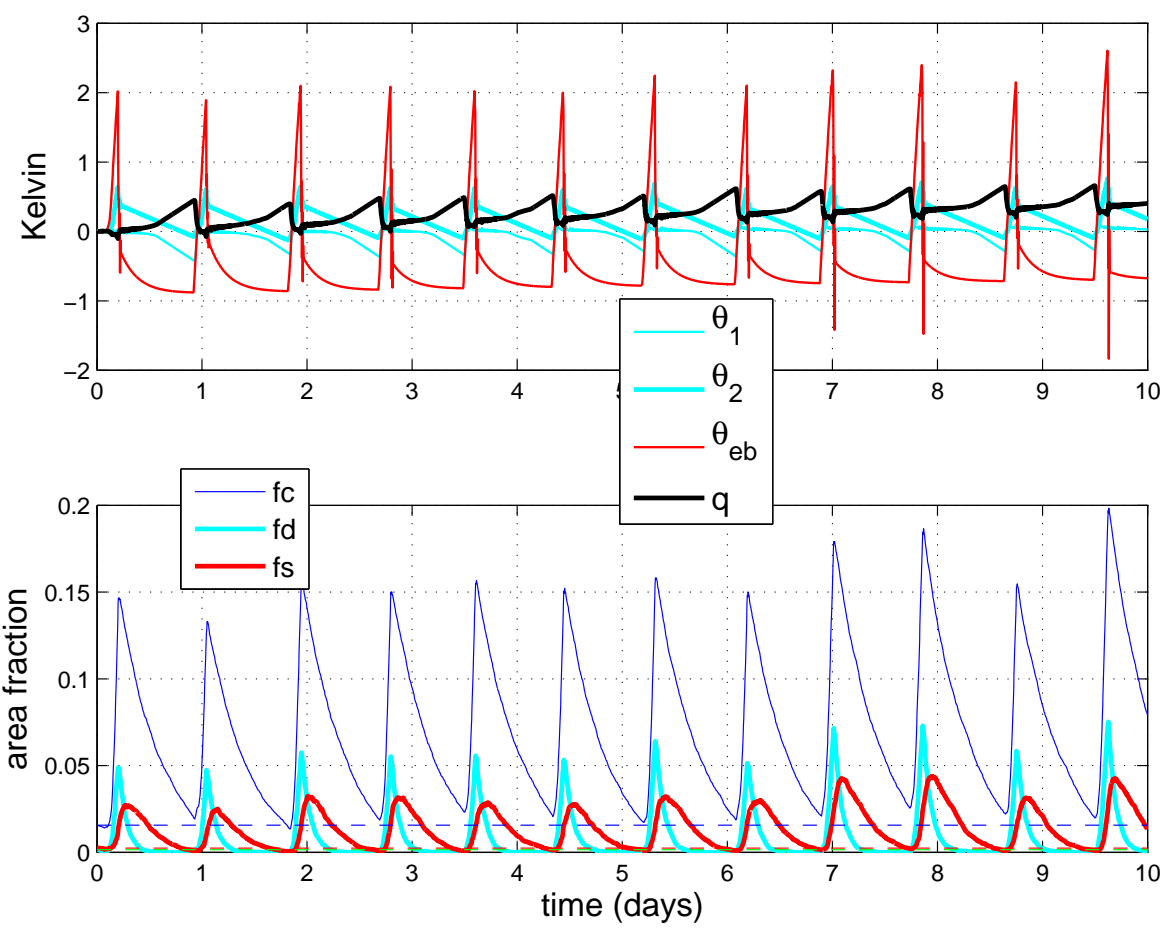

FIG. 5.3. Same as figure 5.1 except for $\gamma_{2}^{\prime}=2$.

where

$$
\begin{aligned}
\mathrm{CAPE} & =\overline{\mathrm{CAPE}}+R\left(\theta_{e b}^{\prime}-\gamma\left(\theta_{1}^{\prime}+\gamma_{2} \theta_{2}^{\prime}\right)\right) \\
\mathrm{CAPE}_{l} & =\overline{\mathrm{CAPE}}+R\left(\theta_{e b}^{\prime}-\gamma\left(\theta_{1}^{\prime}+\gamma_{2}^{\prime} \theta_{2}^{\prime}\right)\right) \quad \text { and } \\
\overline{\mathrm{CAPE}} & =\frac{H_{m}^{2}}{\bar{\alpha}^{2}} \bar{Q}^{2}
\end{aligned}
$$

are the convectively available potential energies integrated over the whole troposphere $(\mathrm{CAPE})$, integrated over the lower troposphere $\left(\mathrm{CAPE}_{l}\right)$ and in radiative-convective equilibrium $(\overline{\mathrm{CAPE}})$. The two different energies, $\mathrm{CAPE}$ and $\mathrm{CAPE}_{l}$ distinguish environments where either deep convection or congestus clouds are active [23, 24]. Typically $\gamma_{2}=0.1$ for deep convective and stratiform heating, which allows only a weak dependence on $\theta_{2}$, while $1 \leq \gamma_{2}^{\prime} \leq 4$ for congestus heating, which emphasizes a strong dependence on $\theta_{2}$ of low level buoyancy. Though we consider both high and low extremes of $\gamma_{2}^{\prime}$ for the sake of comparison, Khouider and Majda [23] used the value $\gamma_{2}^{\prime}=2$, which is computed according to plausible physical assumptions. Here $\bar{\alpha} \approx 15$ $\mathrm{K}$ is the unit scale of temperature and the rest of the model parameters and variables are explained in Table 5.1.

Consistent with the work of Lin and Neelin [45] (see also [46]), the convective time scale $\tau_{c}$ in (5.3) is inversely proportional to the stochastic area fraction of deep convection, and that at equilibrium, when $\sigma_{d}=\bar{\sigma}_{d}, \tau_{c}$ is set to the value $\tau_{c}^{0}=2$ hours used in the original deterministic multicloud model of Khouider and Majda [20, 23].

Clearly, the stochastic-lattice multicloud model affects the large-scale/resolved deterministic equations in (5.1) directly through the area fractions $\sigma_{d}, \sigma_{c}, \sigma_{s}$ in the 

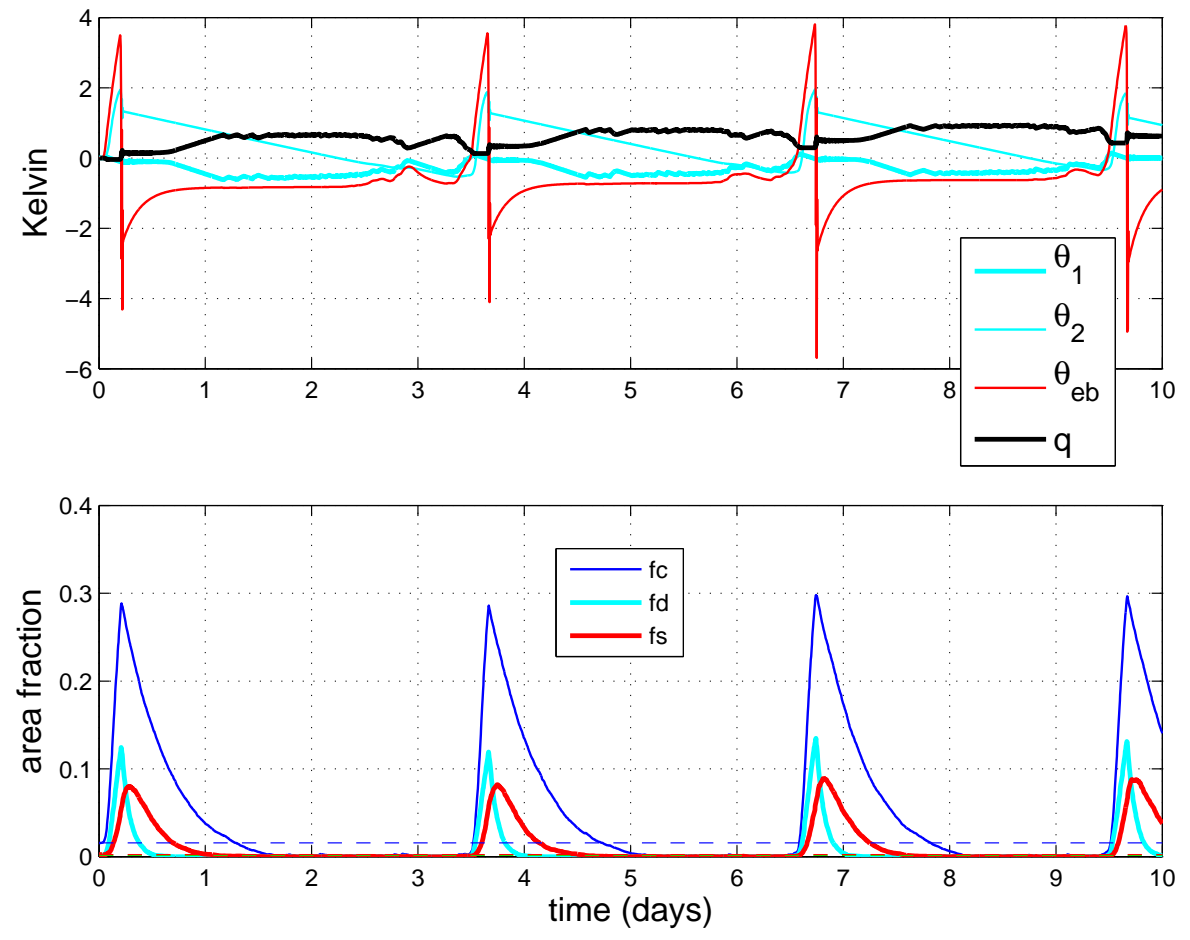

FIG. 5.4. Same as figure 5.1 except for $\gamma_{2}^{\prime}=1$.

definition of the heating rate closures in (5.3). The feedback from the resolved variables to the stochastic subgrid model is accounted for through $C$ and $D$, which as mentioned earlier, are linearly proportional to CAPE and the dryness of the middle of the troposphere

$$
C=\frac{\mathrm{CAPE}}{\mathrm{CAPE}_{0}} \quad \text { and } \quad D=\frac{\theta_{e b}-\theta_{e m}}{T_{0}}
$$

Therefore either (3.1)-(3.7) or (4.1)-(4.7), together with (3.9) and (5.1)-(5.4) form a complete description of the coupled stochastic-deterministic one-column multicloud model, depending on whether we use brute-force Monte-Carlo for the microscopic dynamics or the much-cheaper coarse-grained birth-death process to evolve the stochastic lattice model. In (5.4), $\mathrm{CAPE}_{0}$ and $T_{0}$ are reference values of CAPE and dryness whose values are specified in Tables 5.1 and 5.2.

5.1. A deterministic model using the mean field cloud fraction equations. A dynamical closure for the area fractions is given by the mean field equations (4.8), which, when coupled to equations (5.1)-(5.4) and (3.9) form a fully deterministic system coupling the subgrid area fractions to the large scale convective parametrization. The behavior of this model compared to the stochastic version is discussed below.

A radiative convective equilibrium solution (RCE) for the coupled system is defined as a steady state solution for the large scale equations (5.1) where the area fractions are set to the equilibrium distribution in (3.8) or equivalently to 


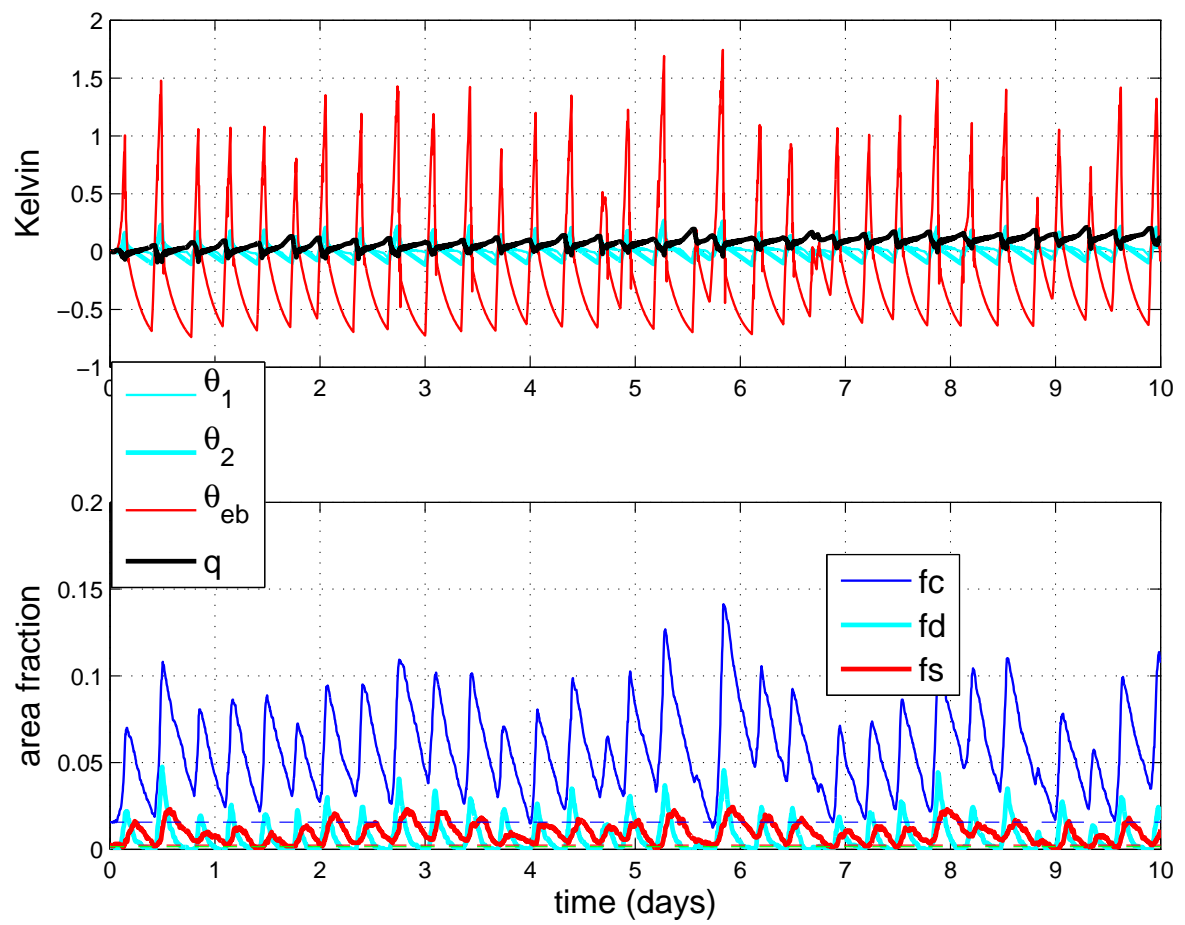

FIG. 5.5. Same as figure 5.1 except for the coarser lattice with $40 \times 40$ sites.

the steady state solution of the mean field equations (4.8). Given the (external) parameters, $\mu, \alpha_{s}, \alpha_{c}, H_{T}, H_{m}, h$, each set of values of the climatological constants $Q_{R, 1}^{0}, \bar{\theta}_{e b}-\bar{\theta}_{e m}, \theta_{e b}^{*}-\bar{\theta}_{e b}$ which yields a unique RCE solution through which the values of $m_{0}, \tau_{e}, \overline{\mathrm{CAPE}}$ and the area fractions, $\bar{\sigma}_{c}, \bar{\sigma}_{d}, \bar{\sigma}_{s}$ are uniquely determined. Given $\overline{\mathrm{CAPE}}$ and $\bar{\theta}_{e b}-\bar{\theta}_{e m}$ the values of the $\bar{\sigma}$ 's are uniquely determined through (3.8) while the value of $\overline{\mathrm{CAPE}}$ itself requires the solution of the nonlinear algebraic equation

$$
Q_{R, 1}^{0}=\bar{\sigma}_{d}(\overline{\mathrm{CAPE}}) \frac{\bar{\alpha}}{H_{m}} \sqrt{\overline{\mathrm{CAPE}}},
$$

which is solved by a numerical root-finding method (f-zero of matlab is used here). In (5.5), $\bar{\sigma}_{d}(\overline{\mathrm{CAPE}})$ highlights the functional dependence of $\bar{\sigma}_{d}$ on $\overline{\mathrm{CAPE}}$ via $(3.8)$ and (5.4). The parameters, $\gamma, \gamma_{2}, \gamma_{2}^{\prime}, \alpha_{2}, a_{1}, a_{2}, a_{0}$ are not involved in the RCE solution, however they, as well as $\alpha_{c}, \alpha_{s}$, and $\mu$, have important effects on the dynamics of the deterministic ODE system in (5.1). These effects are evident even if the area fractions are fixed to their equilibrium values. Unless otherwise stated, the parameter values in Tables 3.1-5.2, are chosen so that the RCE solution for the uncoupled large-scale multicloud parametrization is linearly stable. Slightly raising the values of $\mu$ and/or $\alpha_{c}$, for instance, can lead to instability. The parameter sensitivity of the deterministic one-column model is extensively studied in [21] and therefore it is not repeated here. The important examples are those in which non-trivial dynamics occur in the stochastically coupled system despite the fact that the RCE solution is stable under the deterministic cloud model. Recall from figure 4.1 that the most exciting dynamics associated with the deterministic mean field equations are damped oscilla- 

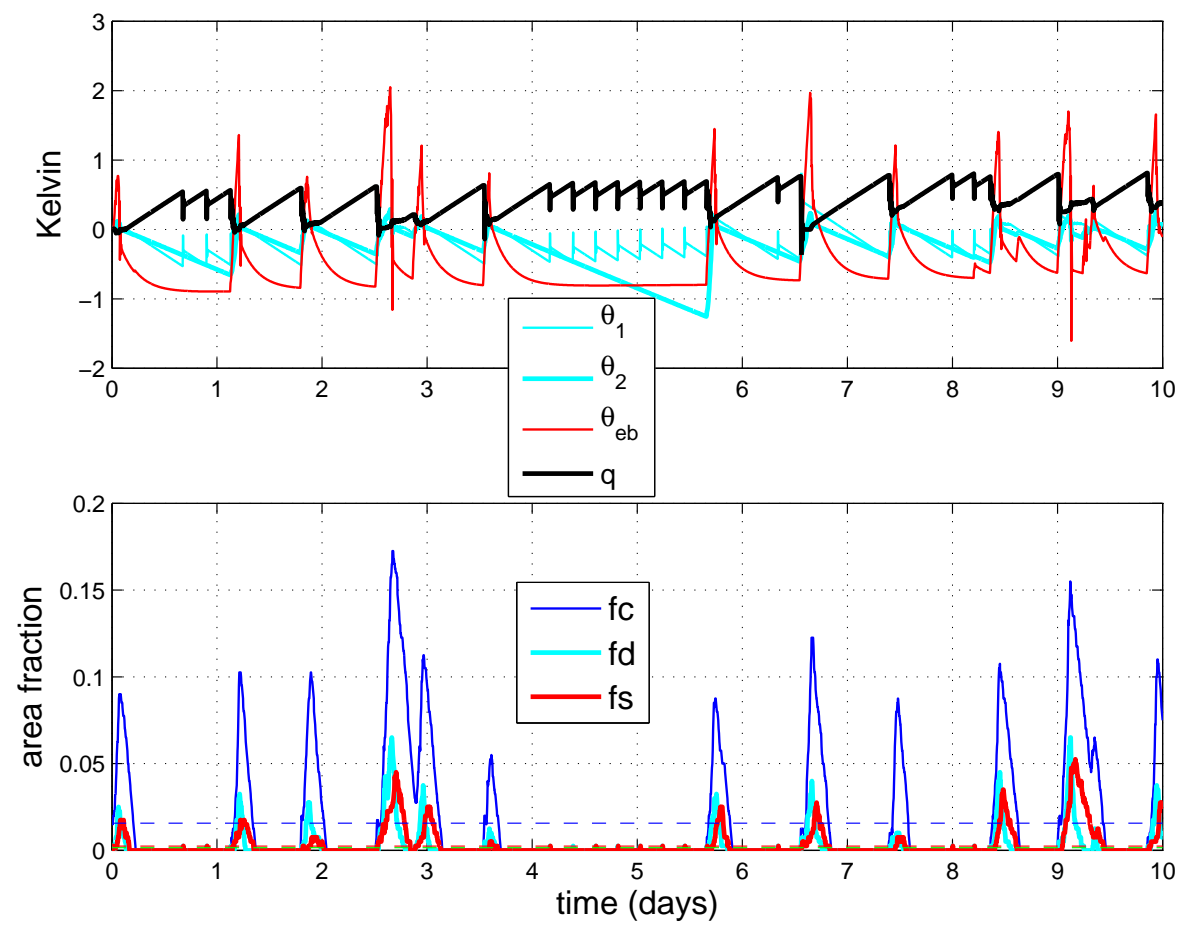

Fig. 5.6. Same as figure 5.1 except for the coarser lattice with $20 \times 20$ sites.

tions. Therefore, coupling the mean field equations to the one-column parametrization equation (5.1) in a parameter regime for which the large scale cloud RCE is a stable equilibrium does not introduce any instability. Though we do not show the results here, numerical simulations confirm that the solutions all damp to their equilibrium values.

5.2. The coupled stochastic model. In this section, we discuss the results of coupling the coarse grained stochastic birth-death system described by the equations in (4.1)-(4.7) and (3.9) to the one-column model equations (5.1). To integrate the equations, we employ a third order Adams-Bashforth scheme in order to minimize both numerical instabilities and artificial dissipation. The coarse grained birth-death process is evolved in time by means of an acceptance-rejection Markov Chain Monte Carlo method based on Gillespie's exact algorithm $[5,6]$ where a rescaled version of the transition rates in (3.5)-(3.7) is utilized, consistent with the approximations in (4.1)-(4.7). We evolve $N=100 \times 100$ lattice sites of the coarse grained system with much less computational expense than would be necessary using the full Monte Carlo dynamics.

Below, we present some results of the numerical simulations for the coupled stochastic-deterministic system for the parameter values in Tables 3.1-5.2. The results are sensitive to variations in most of the model parameters listed in Table 5.1 and to the cloud time scales in Table 3.1. Here we consider only the two main cases reported in Table 5.2, with the corresponding stochastic time scales reported in Table 3.1 in order to showcase some of the most interesting and typical regimes of the 


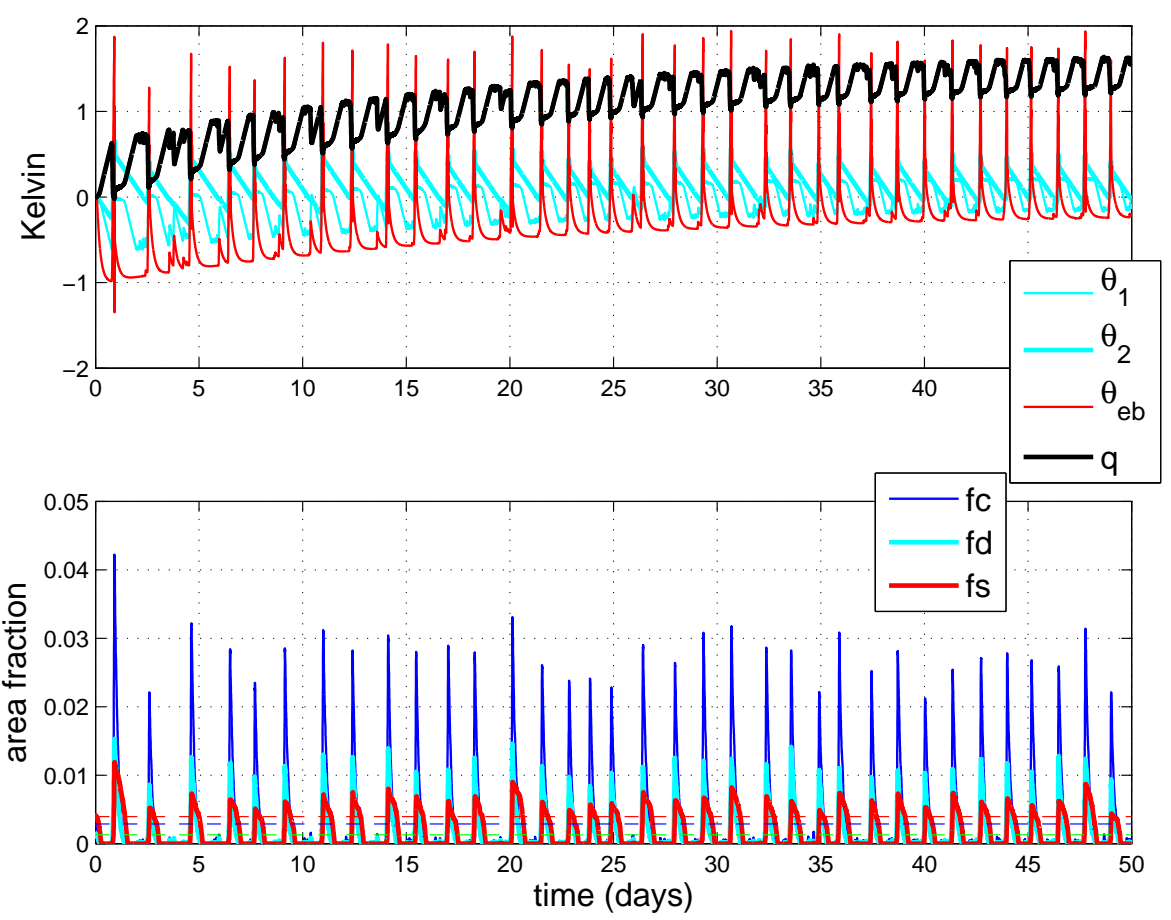

Fig. 5.7. Same as figure 5.1 except for the parameter values corresponding to Case 2 column in Table 5.2: $C A P E_{0}=2000 \mathrm{~J} / \mathrm{kg}$.

coupled deterministic/stochastic model; a more thorough parameter sensitivity study is necessary and is deferred to future work.

A time series of both the large-scale variables (top) and the cloud area-fraction coverages for the parameter values in Table 5.1 and on the first rows (case 1) of Table 3.1 and 5.2, are plotted in figure 5.1 and in greater detail in figure 5.2. Most notable is the time synchronization of the oscillations of both the stochastic and the deterministic variables. After the transient period of less than one day, the time series enter a quasi-periodic oscillatory regime with roughly a half day period. The other striking feature is the organization of the cloud fraction oscillations. Congestus bursts are followed by deep convective busts, which in turn lead stratiform peaks, consistent with the physical intuition utilized to design the model. The fluctuations in the cloud area fractions are directly related to changes in the large scale fields. The increase in congestus area fraction is a direct response to CAPE build up (corresponding to the $\theta_{e b}$-peaks). Congestus heating anomalies then yield a rise in $\theta_{2}$ by direct heating, which yields a rise in $\theta_{e m}$ - an artificial moistening, which in turn triggers the peak in $\sigma_{d}$ and the rise in $\sigma_{s}$ follows naturally as expected, since there is non-zero probability that a fraction of the deep convective clouds are converted into stratiform clouds, regardless of the background state. Notice that the moisture anomalies are weak in this case although some regeneration of moisture follows quickly after a deep convective sudden drying, due to the evaporation of stratiform rain.

In figure 5.3, the same experiment is repeated but with the more realistic, smaller value $\gamma_{2}^{\prime}=2$. The most considerable change is seen in the period of the oscillations 


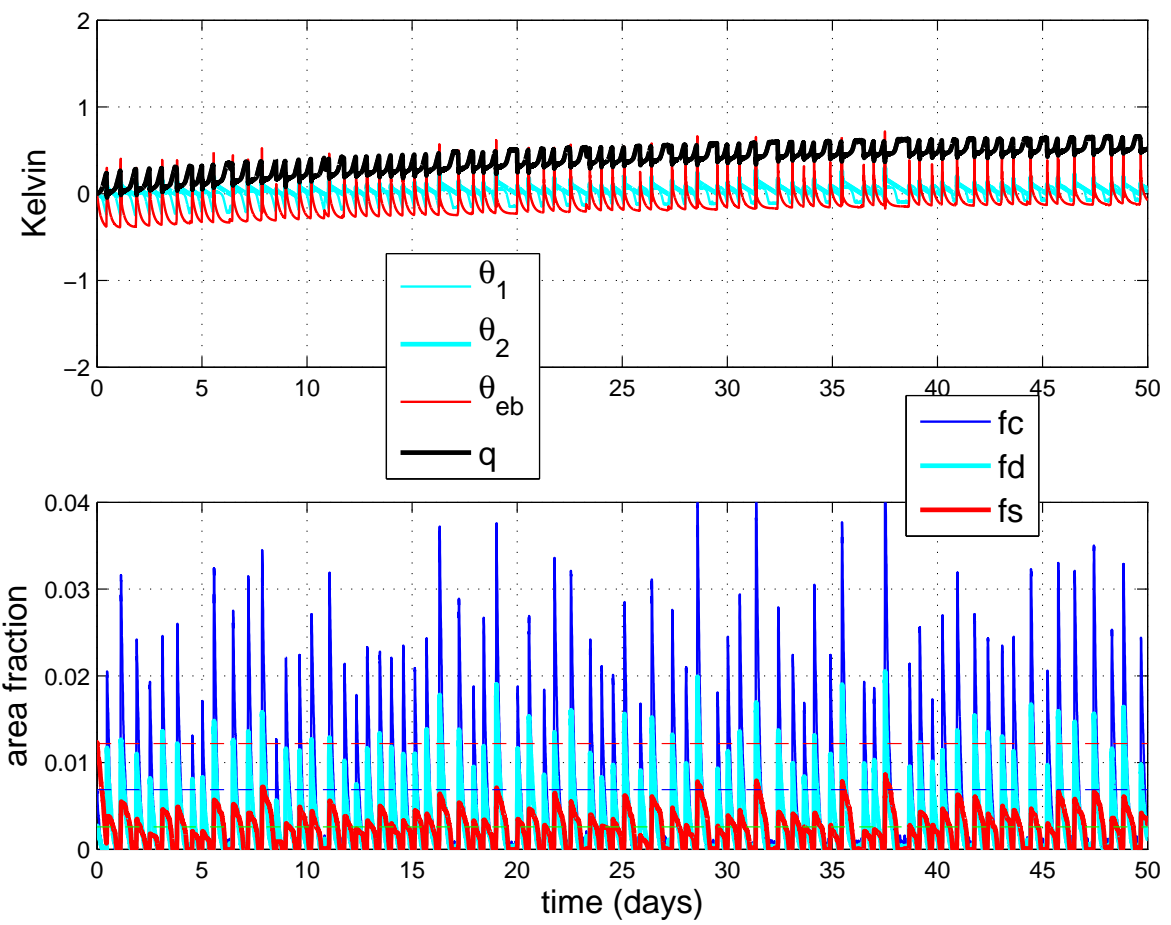

FIG. 5.8. Same as figure 5.7 except for the low value $C A P E_{0}=200 \mathrm{~J} / \mathrm{kg}$.

which increase from a half day to about one day. Recall that $\gamma_{2}^{\prime}$ directly affects the way in which low level CAPE depends on low-level potential temperature anomalies, i.e, $\theta_{2}$. A large $\gamma_{2}^{\prime}$ implies a rapid heating of the low-level troposphere by congestus convection and a quick recovery in $\theta_{2}$ during the congestus episode, which then leads to a decrease in $\theta_{e b}-\theta_{e m}$ (an artificial moistening) creating deep convective clouds, and so on. On the other hand when $\gamma_{2}^{\prime}=2$, the congestus heating is not artificially large and therefore doesn't yield artificially high $\theta_{2}$ values, allowing some time for realistic moistening, i.e, a significant rise in mid-tropospheric moisture, $q$, prior to deep convection. The other extreme case is presented in figure 5.4 for the value $\gamma_{2}^{\prime}=1$. As one would expect from the previous two cases, the period of oscillation increases significantly to about three days. Consequently, convective activity completely ceases between the intermittent bursts. During the quiet episodes, moisture rises by about $1 \mathrm{~K}$ and may play a central role in the regeneration of deep convection.

We emphasize that the regeneration of CAPE, marked by the sudden increase in $\theta_{e b}$ by a few degrees over a few hours, is consistent with the relaxation time of $\tau_{e} \approx 8$ hours associated with surface evaporation. If downdrafts are completely absent and $\theta_{e b}$ is initially set to its equilibrium value, so that $\theta_{e b}^{*}-\theta_{e b}(0)=10 \mathrm{~K}$ according to the RCE solution, the parameters allow an increase in $\theta_{e b}$ of about $20 \mathrm{~K}$ over a period of 8 hours. This exponential growth of $\theta_{e b}$ is evident from the higher resolution plots; in figure $5.2, \theta_{e b}$ increases about $1.5 \mathrm{~K}$ over a period of about 1.5 hours and similar scale of growth is evident in the more intermittent case of figure 5.4.

We address the sensitivity of the model to the number of lattice sites in figures 


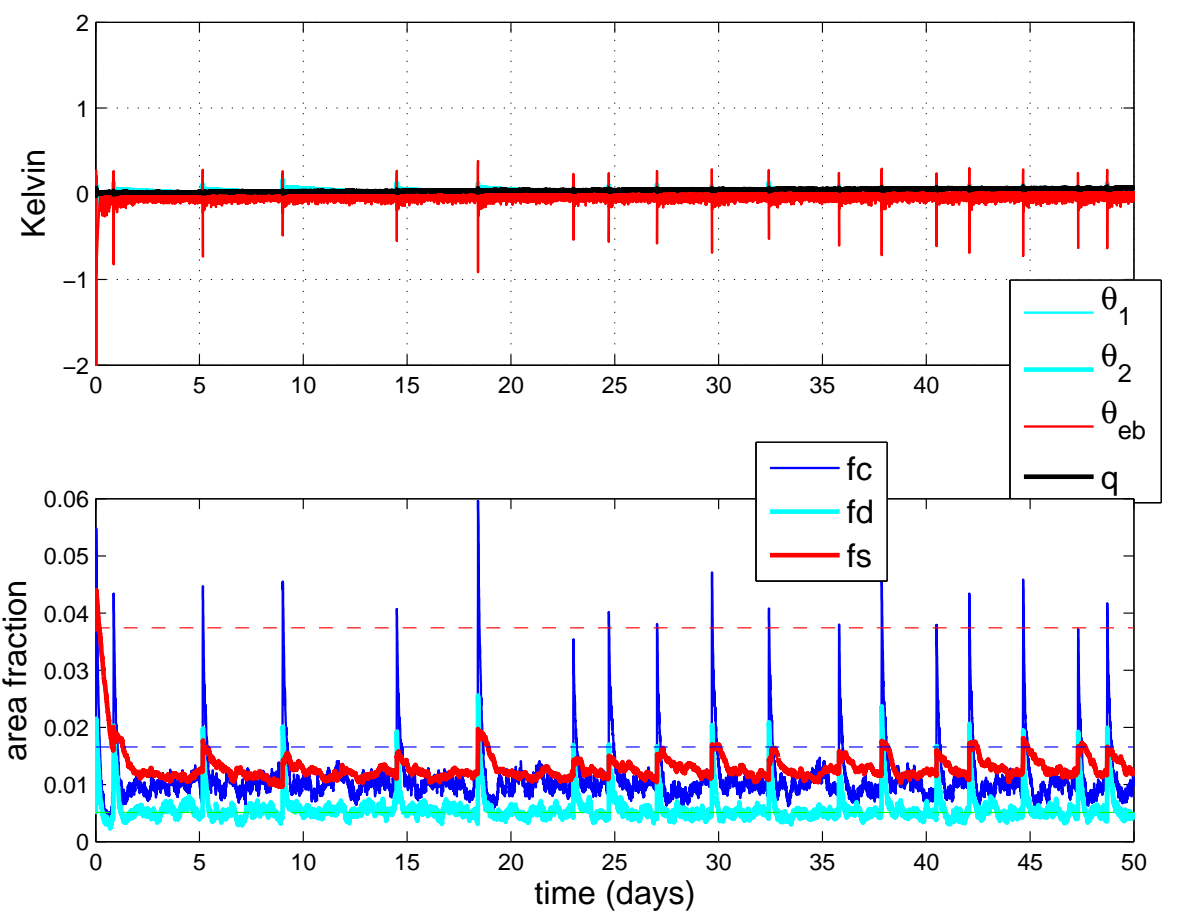

FIG. 5.9. Same as figure 5.7 except for the low value $C A P E_{0}=20 \mathrm{~J} / \mathrm{kg}$.

5.5 and 5.6 where $40 \times 40$ and $20 \times 20$ sites are used, respectively, as opposed to the $100 \times 100$ sites used elsewhere in this paper. The means and standard deviations of each one of the dynamical variables for the three different values of site numbers are reported in Table 5.3. As one would expect decreasing the number of lattice elements causes an overall increase in the variability of the stochastic area fractions, $f_{c}, f_{s}$. Correspondingly, the variability of the large scale variables, $\theta_{1}, \theta_{2}, q$, increases, as revealed by the standard deviations in Table 5.3. The boundary layer equivalent potential temperature, $\theta_{e b}$, whose variability is dominated by the deterministic surface evaporation process, is more robust against changes in the number of lattice sites. Consistent with the balance between deep convection and boundary layer fluxes, variations in standard deviation of deep convective clouds follow that of $\theta_{e b}$. However, it is important to note, from Table 5.3, the consistent decrease of the mean cloud area fractions and the changes in mean large scale quantities as the number of lattice sites is decreased from $100 \times 100$ to $40 \times 40$ to $20 \times 20$. A coarser lattice tends to lead to a colder and drier boundary layer but significantly colder and moister middle troposphere that compensate for the small cloud fractions. The most noticeable feature when comparing figures 5.5 and 5.6 is that as the number of lattice sites is reduced to $20 \times 20$ the system becomes much more intermittent and quiescent periods can last up to two days.

Figures 5.7, 5.8, and 5.9 correspond to Case 2 in Table 3.1, and 5.2 for $\mathrm{CAPE}_{0}=2000,200$ and $20 \mathrm{~J} / \mathrm{kg}$, respectively. The long integration time shows a slow upward drift of moisture above its RCE value, which takes place during the first 10-20 days. This is especially evident for the case $\mathrm{CAPE}_{0}=2000 \mathrm{~J} / \mathrm{kg}$ in figure 5.7 


\begin{tabular}{|c|c|c|c|}
\hline Parameter & Description & Nature & Value \\
\hline$Q_{R, 1}^{0}$ & $\begin{array}{l}\text { First baroclinic radiative } \\
\text { cooling }\end{array}$ & Climatological & $1 \mathrm{~K} \mathrm{day}^{-1}$ \\
\hline$Q_{R, 2}^{0}$ & $\begin{array}{l}\text { Second baroclinic radia- } \\
\text { tive cooling }\end{array}$ & Determined at RCE & Varies \\
\hline $\bar{\theta}_{e b}-\bar{\theta}_{e m}$ & \begin{tabular}{lr} 
Discrepancy & \multicolumn{2}{r}{ between } \\
boundary & layer and \\
middle & tropospheric \\
equivalent & potential \\
temperatures &
\end{tabular} & Climatological & $11 \mathrm{~K}$ \\
\hline$\theta_{e b}^{*}-\bar{\theta}_{e b}$ & $\begin{array}{l}\text { Discrepancy between sat- } \\
\text { uration and actual bound- } \\
\text { ary layer equivalent po- } \\
\text { tential temperatures }\end{array}$ & Climatological & $10 \mathrm{~K}$ \\
\hline$\tau_{c}^{0}$ & $\begin{array}{l}\text { Reference convective time } \\
\text { scale }\end{array}$ & Fixed parameter & 2 hours \\
\hline$m_{0}$ & Downdraft mass flux scale & Determined at RCE & Varies \\
\hline$\tau_{e}$ & $\begin{array}{l}\text { Sea surface evaporation } \\
\text { time scale }\end{array}$ & Determined at RCE & Varies \\
\hline$\overline{\mathrm{CAPE}}$ & Value of CAPE at RCE & Determined at RCE & Varies \\
\hline$\mu$ & $\begin{array}{l}\text { Relative contribution of } \\
\text { stratiform evaporative } \\
\text { cooling to downdrafts }\end{array}$ & Tunable & Varies $0.25,0.5$ \\
\hline$\alpha_{s}$ & $\begin{array}{l}\text { Contribution of CAPE to } \\
\text { stratiform heating }\end{array}$ & Fixed & 0.25 \\
\hline$\alpha_{c}$ & $\begin{array}{l}\text { Contribution of CAPE to } \\
\text { congestus heating }\end{array}$ & Tunable & $0.1-0.5$ \\
\hline
\end{tabular}

TABLE 5.1. Constants and parameters for the deterministic multicloud large-scale parametrization.

but much less significant for the case $\mathrm{CAPE}_{0}=200 \mathrm{~J} / \mathrm{kg}$ in figure 5.8 and nonexistent when $\mathrm{CAPE}_{0}=20 \mathrm{~J} / \mathrm{kg}$ in figure 5.9 .

Note that $\mathrm{CAPE}_{0}$ acts as an "activation-energy" threshold for convection, and these results suggest that when this threshold is low, moisture anomalies tend to be small and when it is large, moisture anomalies tend to be large. A plausible explanation for this correlation resides in the way CAPE and moisture affect the cloud transition rates in (3.6) and (3.7). When $\mathrm{CAPE}_{0}$ is large, the cloud formation time scales (i.e, $\left.\tau_{0 i}, i=1,2,3\right)$ tend to be large, allowing enough time for large scale moistening (by downdrafts) to take place between successive convective episodes which, in turn, allow large scale precipitation to provide a significant amount of drying. On the other hand, small values of $\mathrm{CAPE}_{0}$ yield short cloud formation timescales and therefore result in rapid and small fluctuations of moisture and more intermittency.

\section{Summary and discussion}

A stochastic lattice model to account for the unresolved variability due to organized tropical convection in general circulation models is presented here. It is based on the assumption that organized convection involves three cloud types, congestus, deep, and stratiform, which occur in a more or less organized manner in space and time. Each lattice site is represented by a Markov process taking discrete values from 
Table 5.1 (continued)

\begin{tabular}{|c|c|c|c|}
\hline$a_{1}$ & $\begin{array}{l}\text { Contribution of } \theta_{e b} \text { to } \\
\text { deep convective heating } \\
\text { anomalies }\end{array}$ & Fixed & 0.1 \\
\hline$a_{2}$ & $\begin{array}{l}\text { Contribution of } q \text { to deep } \\
\text { convective heating anoma- } \\
\text { lies }\end{array}$ & Fixed & 0.9 \\
\hline$a_{0}$ & $\begin{array}{l}\text { Contribution of } \theta_{1} \text { to deep } \\
\text { convective heating anoma- } \\
\text { lies }\end{array}$ & Fixed & 5 \\
\hline$\gamma_{2}$ & $\begin{array}{l}\text { Relative contribution of } \theta_{2} \\
\text { to deep convective heating } \\
\text { anomalies }\end{array}$ & Fixed & 0.1 \\
\hline$\gamma$ & $\begin{array}{l}\text { Contribution of } \theta_{1} \text { to } \\
\text { CAPE anomalies }\end{array}$ & Fixed & 1.7 \\
\hline$R$ & CAPE constant & Fixed & $2.1413 \mathrm{e}-04 \mathrm{~J} / \mathrm{kg} \mathrm{K}^{-1}$ \\
\hline$\gamma_{2}^{\prime}$ & $\begin{array}{l}\text { Relative contribution of } \theta_{2} \\
\text { to low level CAPE anoma- } \\
\text { lies }\end{array}$ & Tunable & $2-4$ \\
\hline $\mathrm{CAPE}_{0}$ & $\begin{array}{l}\text { Reference value of CAPE } \\
\text { used in stochastic model }\end{array}$ & Changes & $20-2000 \mathrm{~J} / \mathrm{Kg}$ \\
\hline$T_{0}$ & $\begin{array}{l}\text { Reference value of dryness } \\
\text { used in stochastic model }\end{array}$ & Tunable & $15 \mathrm{~K}$ \\
\hline
\end{tabular}

\begin{tabular}{c|cc}
\hline Case & 1 & 2 \\
\hline $\mathrm{CAPE}_{0}$ & $2000 \mathrm{~J} / \mathrm{kg}$ & $2000 \mathrm{~J} / \mathrm{kg}, 200 \mathrm{~J} / \mathrm{kg}, 20 \mathrm{~J} / \mathrm{kg}$ \\
$\mu$ & 0.25 & 0.5 \\
$\gamma_{2}^{\prime}$ & $4,2,1$ & 2 \\
$\alpha_{c}$ & 0.1 & 0.5 \\
$\mathrm{CAPE}$ & $6.5087 \mathrm{~J} / \mathrm{kg}$ & $8.7366 \mathrm{~J} / \mathrm{kg}, 2.1426 \mathrm{~J} / \mathrm{kg}$ \\
$\tau_{e}$ & $8.33 \mathrm{hours}$ & - \\
$m_{0}$ & $0.0181 \mathrm{~m} \mathrm{~s}^{-1}$ & $0.0184 \mathrm{~m} \mathrm{~s}^{-1}, 0.0164 \mathrm{~m} \mathrm{~s}^{-1}$ \\
$Q_{R, 2}^{0}$ & $0.6421 \mathrm{~K} \mathrm{day}^{-1}$ & $0.3547 \mathrm{~K} \mathrm{day}^{-1}, 0.1523 \mathrm{~K} \mathrm{day}^{-1}$ \\
$\tau_{l k}$ 's & Tab. 1, Case 1 & Tab. 1, Case 2 \\
$R_{23}$ & equation $(3.6)$ & equation $(3.7)$ \\
\hline
\end{tabular}

TABLE 5.2. Parameter values used in each one of the different cases considered for coupled numerical simulations.

0 to 3 according to whether the sky is clear or a cloud of a certain type is active at that site. The transition from one state to another occurs according to some probability laws motivated by physical intuition gained (elsewhere) from observations and detailed numerical simulations of tropical convective systems. The transition time scales depend, in a physically realistic way, on the large scale distribution of CAPE and dryness of the middle troposphere. For the very different physical processes of upscale convective momentum transport, Majda and Stechmann [37] have developed a class of stochastic models based on similar design principles. When CAPE is positive and the atmosphere is dry congestus clouds are formed with high probability and when CAPE is positive and the atmosphere is moist congestus sites are converted into 


\begin{tabular}{cccc} 
number of sites & $100 \times 100$ & $40 \times 40$ & $20 \times 20$ \\
\hline mean & 0.0208 & 0.0087 & -0.1497 \\
$\theta_{1}$ & -0.0117 & -0.0040 & -0.2245 \\
$\theta_{2}$ & -0.1831 & -0.1701 & -0.4311 \\
$\theta_{e b}$ & 0.0738 & 0.0773 & 0.4152 \\
$q$ & 0.0810 & 0.0631 & 0.0185 \\
$f_{c}$ & 0.0088 & 0.0076 & 0.0031 \\
$f_{d}$ & 0.0142 & 0.0105 & 0.0035 \\
$f_{s}$ & & & \\
$\theta_{1}$ & 0.0137 & 0.0266 & 0.1882 \\
$\theta_{2}$ & 0.0692 & 0.0672 & 0.2322 \\
$\theta_{e b}$ & 0.4994 & 0.4954 & 0.4667 \\
$q$ & 0.0427 & 0.0443 & 0.1956 \\
$f_{c}$ & 0.0221 & 0.0271 & 0.0324 \\
$f_{d}$ & 0.0091 & 0.0095 & 0.0081 \\
$f_{s}$ & 0.0050 & 0.0063 & 0.0069 \\
\hline
\end{tabular}

TABLE 5.3. Change in variability with respect to changes in number of lattice sites.

deep convective sites with high probability. Deep convective sites are then converted into stratiform sites in a manner which may or may not depend on the background CAPE.

For the sake of simplicity the individual lattice sites are assumed to be independent of each other so that a coarse-grained stochastic birth-death system for the cloud area fraction of each cloud type is easily derived and numerically integrated with very low computational overhead. The birth-death system naturally yields a system of mean-field linear ordinary differential equations whose equilibrium solution corresponds to the stationary distribution of the Markov chains. After a short transient period, the stochastic system oscillates around this equilibrium solution forever, when the large scale dynamics are frozen.

As expected, the dynamics of the stochastic lattice model are sensitive to both the prescribed convective time scales and variations in the large-scale external variables. For instance, the mean-field equilibrium is in a purely damped mode when CAPE is small, and bifurcates to an oscillatory damped regime for large enough values of CAPE. As the numerics demonstrate, this has the non-trivial implication that, when CAPE is large the stochastic oscillations around the equilibrium state are rapid and have large amplitudes, whereas when CAPE is small the oscillations have small amplitudes with very intermittent large excursions. This is somewhat similar to what is observed in Katsoulakis et al. [13] and in Majda et al. [36].

The stochastic multicloud model is coupled to a deterministic tropical climate model consisting of a simple ODE system representing the dynamics of a twobaroclinic model over one GCM grid-box: a one-column model, based on the multicloud model equations of Khouider and Majda [20, 24]. The multicloud deterministic equations are integrated by a 3rd order Adams-Bashforth method while the stochastic birth-death cloud model is evolved according to the exact stochastic algorithm of Gillespie $[5,6]$, where the cloud populations are viewed as a sum of Poisson processes.

An important result of this paper is that the stochastic multicloud model responds 
to variations in the large scale variables according to the intuitive rules used to construct these models. Quasi-periodic fluctuations in CAPE and moisture are correlated with well organized large oscillations in the cloud area fractions in a kind of "stochastic resonance" behavior. Positive $\theta_{e b}$ anomalies (positive CAPE) trigger a rise in congestus cloudiness which preconditions the environment and provokes deep convective bursts. Deep convection is followed by stratiform convection, which consumes CAPE by downdrafts and helps moisten the environment for the next convective episode. A detailed understanding of the complexity with which the coupled system responds to changes in the various parameters utilized here (and listed in Tables 3.1 and 5.1) is beyond the scope of this paper. However, the few simulations reported at the end of section 5 demonstrate two important facts.

1. The time scale with which congestus heating responds to lower tropospheric temperature (i.e $\theta_{2}$ ) is crucial for setting the frequency and strength of the stochastic oscillations of the cloud area fractions and, therefore, of the large scale dynamics. Large congestus-to- $\theta_{2}$ time-scales (corresponding to small $\gamma_{2}^{\prime}$ ) yield slow but somewhat strong oscillations in both the large scale variables and the cloud area fractions while small congestus-to- $\theta_{2}$ time-scales yield rapid and somewhat weaker oscillations. The average oscillation period is about 3 days when $\gamma_{2}^{\prime}=1$ and half day when $\gamma_{2}^{\prime}=4$. Coincidentally, the more realistic value of $\gamma_{2}^{\prime}=2$, yields a one-day period.

2. $\mathrm{CAPE}_{0}$, the reference scale by which the stochastic cloud model responds to variations in large scale CAPE, acts as an "activation energy", yielding strong and more intermittent bursts of convection when $\mathrm{CAPE}_{0}$ is large $(2000 \mathrm{~J} / \mathrm{kg}$ ) and very rapid and weak fluctuations in both the cloud area fractions and large scale climate variables when $\mathrm{CAPE}_{0}$ is small $(200 \mathrm{~J} / \mathrm{kg}$ and more so when $20 \mathrm{~J} / \mathrm{kg}$ ). Interestingly however, the weaker value of $20 \mathrm{~J} / \mathrm{kg}$ seems to present very intermittent strong bursts, from time-to-time, on top of the very weak, faster oscillation.

Acknowledgement. The work of B.K. is sponsored in part by a grant from the Natural Sciences and Engineering Research Council of Canada and the Canadian Foundation for Climate and Atmospheric Sciences. J.A.B. is supported by NSF DMS0604947. A.J.M. is partly supported by the National Science Foundation and the Office of Naval Research. Comments by the anonymous referee that led to some clarifications and improvement of the text are highly acknowledged.

\section{REFERENCES}

[1] R. Buizza, M. Miller and T.N. Palmer, Stochastic representation of model uncertainties in the ECWMF Ensemble Prediction System, Q.J.R. Meteorol. Soc., 125, 2887-2908, 1999.

[2] D. Crommelin and E. Vanden Eijden, Subgrid scale parametrization with conditional Markov chains, J. Atmos. Sci., 65, 2661-2675, 2008.

[3] T.J. Dunkerton and F.X. Crum, Eastward propagating 2- to 15-day equatorial convection and its relation to the tropical intraseasonal oscillation, J. Geophys. Res., 100, 25781-25790, 1995.

[4] K.A. Emanual, Atmospheric Convection, Oxford, 1994.

[5] D.T. Gillespie, An exact method for numerically simulating the stochastic coalescence process in a cloud, J. Atmos. Sci., 32, 1977-1989, 1975.

[6] D.T. Gillespie, Exact stochastic simulation of coupled chemical reactions, J. Phys. Chem., 81, 2341-2361, 1977.

[7] H. Hendon and B. Liebmann, Organization of convection within the Madden-Julian Oscillation, J. Geophys. Res., 99, 8073-8083, 1994. 
[8] D.J. Horntrop, M.A. Katsoulakis and D.G. Vlachos, Spectral methods for mesoscopic models of pattern formation, J. Comp. Phys., 173, 361-390, 2001.

[9] R.A. Houze, Stratiform precipitation in regions of convection: a meteorological paradox? Bull. Amer. Meteor. Soc., 78, 2179-2196, 1997.

[10] R.H. Johnson, T.M. Rickenbach, S.A. Rutledge, P.E. Ciesielski and W.H. Schubert, Trimodal characteristics of tropical convection, J. Climate, 12, 2397-2407, 1999.

[11] M.A. Katsoulakis, A.J. Majda and A. Sopasakis, Multiscale coupling in prototype hybrid deterministic/stochastic systems. Part I: deterministic closures, Commun. Math. Sci., 2, 255-294, 2004.

[12] M.A. Katsoulakis, A.J. Majda and A. Sopasakis, Multiscale coupling in prototype hybrid deterministic/stochastic systems. Part II: stochastic closures, Commun. Math. Sci., 3, 453-478, 2005.

[13] M.A. Katsoulakis, A.J. Majda and A. Sopasakis, Intermittency, metastability, and coarse graining for coupled deterministic stochastic lattice systems, Nonlinearity, 19, 1021-1047, 2006.

[14] M.A. Katsoulakis, A.J. Majda and A. Sopasakis, Prototype hybrid couplings of macroscopic deterministic models and microscopic stochastic lattice dynamics, Contemporary Mathematics, 429, 143-187, 2007.

[15] M.A. Katsoulakis and D.G. Vlachos, From microscopic interactions to macroscopic laws of cluster evolution, Phys. Rev. Lett., 84, 1511-1514, 2000.

[16] M.A. Katsoulakis and P.E. Souganidis, Stochastic Ising models and anisotropic front propagation, J. Stat. Phys., 87, 63-89, 1997.

[17] M.A. Katsoulakis and D.G. Vlachos, Hierarchical kinetic Monte Carlo simulations for diffusion of interacting molecules, J. Chem. Phys., 112, 9412-9427, 2003.

[18] M.A. Katsoulakis, A.J. Majda and G.D. Vlachos, Coarse-grained stochastic processes for microscopic lattice systems, Proc. Nat. Acad. Sci. USA, 100, 782-787, 2003.

[19] M.A. Katsoulakis, A.J. Majda and G.D. Vlachos, Coarse-grained stochastic processes and Monte Carlo simulations in lattice systems, J. Comp. Phys., 186, 250-278, 2003.

[20] B. Khouider and A.J. Majda, A simple multicloud parametrization for convectively coupled tropical waves. Part I: linear analysis, J. Atmos. Sci, 63, 1308-1323, 2006.

[21] B. Khouider and A.J. Majda, Model multicloud parametrizations with crude vertical structure, Theoretical and Computational Fluid Dynamics, Special Issue: Theoretical developments in tropical meteorology, 20, 351-375, 2006.

[22] B. Khouider and A.J. Majda, A simple multicloud parametrization for convectively coupled tropical waves. Part II: nonlinear simulations, J. Atmos. Sci., 64, 381-400, 2007.

[23] B. Khouider and A.J. Majda, Multicloud models for tropical convection: enhanced congestus heating, J. Atmos. Sci., 65, 895-914, 2008.

[24] B. Khouider and A.J. Majda, Equatorial convectively coupled waves in a simple multicloud model, J. Atmos. Sci., 65, 3376-3397, 2008.

[25] B. Khouider, A.J. Majda and M. Katsoulakis, Coarse grained stochastic models for tropical convection, Proc. Nat. Acad. Sci. USA, 100, 11941-11946, 2003.

[26] G.N. Kiladis, K.H. Straub and P. Haertl, Zonal and vertical structure of the Madden-Julian oscillation, J. Atmos. Sci., 62, 2790-2809, 2005.

[27] G.N. Kiladis, M.C. Wheeler, P.T. Haertel, K.H. Straub and P.E. Roundy, Convectively coupled equatorial waves, Reviews of Geophysics, 47, RG2003, doi:1029/2008R000266, 2008.

[28] G. Lawler, Introduction to stochastic processes, Chapman \& Hall/CRC, 192, 1995.

[29] X. Lin and R.H. Johnson, Kinematic and thermodynamic characteristics of the flow over the Western Pacific Warm Pool during TOGA COARE, J. Atmos. Sci., 53, 695-715, 1996.

[30] R. Madden and P. Julian, Description of global scale circulation cells in tropics with a 40-50 day period, J. Atmos. Sci., 29, 1109-1123, 1972.

[31] R.A. Madden and P. Julian, Observations of the 40-50 day tropical oscillation - a review, Mon. Weather Rev., 122, 814-837, 1994.

[32] A.J. Majda, B. Khouider, G.N. Kiladis, K.H. Straub and M.G. Shefter, A model for convectively coupled tropical waves: Nonlinearity, rotation, and comparison with observations, J. Atmos. Sci., 61, 2188-2205, 2004.

[33] A. Majda and M. Shefter, Waves and instabilities for model tropical convective parametrizations, J. Atmos. Sci., 58, 896-914, 2001.

[34] A.J. Majda, New multiscale models and self-similarity in tropical convection, J. Atmos. Sci., 1393-1404, 2007.

[35] A.J. Majda and B. Khouider, Stochastic and mesoscopic models for tropical convection, Proc. Nat. Acad. Sci., 99, 1123-1128, 2002.

[36] A.J. Majda, C. Franzke and B. Khouider, An applied mathematics perspective on stochastic modelling for climate, Phil. Trans. Roy. Soc. A, 366, 2429-2455, 2008. 
[37] A.J. Majda and S. Stechmann, Stochastic models for convective momentum transport, Proc. Nat. Acad. Sci., 105, 17614-17619, 2008.

[38] A.J. Majda, S. Stechmann, and B. Khouider, An MJO analog and intraseasonal variability in a multi-cloud model above the equator, Proc. Nat. Acad. Sci. USA, 104, 9919-9924, 2007.

[39] B.E. Mapes, Convective inhibition, subgridscale triggering energy, and "stratiform instability" in a toy tropical wave model, J. Atmos. Sci., 57, 1515-1535, 2000.

[40] B.E. Mapes, S.N. Tulich, J. Lin and P. Zuidema, The mesoscale convection life cycle: building block or prototype for large-scale tropical waves? Dyn. Atmos. Oceans., Special Issue: Atmospheric Convection and Wave Interactions: Convective Life Cycles and Scale Interactions in Tropical Waves, 42, 3-29, 2006.

[41] M.W. Moncrieff and C. Liu, Representing convective organization in prediction models by a hybrid strategy, J. Atmos. Sci., 63, 3404-3420, 2006.

[42] M.W. Moncrieff, Organized convective systems: Archetypal dynamical models, mass and momentum flux theory, and parametrization, Quart. J. Roy. Meteorol. Soc., 118, 819-850, 1992.

[43] M.W. Moncrieff, Analytic representation of the large-scale organization of tropical convection, J. Atmos. Sci., 61, 1521-1538, 2004.

[44] T. Nakazawa, Tropical super clusters within intraseasonal variations over the western Pacific, J. Meteor. Soc. Japan, 66, 823-839, 1988.

[45] J.W.B. Lin and D. Neelin, Toward stochastic deep convective parameterization in general circulation models, Geophy. Res. Lett., 27, 3691-3694, 2000.

[46] J.W.B. Lin and D. Neelin, Influence of a stochastic moist convective parameterization on tropical climate variability, Geophys. Res. Lett., 30, 1162-1165, 2003.

[47] J.M. Slingo and 21 co-authors, Intraseasonal oscillation in 15 atmospheric general circulation models: results from an AMIP diagnostic subproject, Climate Dyn., 12, 325-357, 1996.

[48] K.H. Straub and G.N. Kiladis, The observed structure of convectively coupled Kelvin waves: comparison with simple models of coupled wave instability, J. Atmos. Sci., 60, 1655-1668, 2003.

[49] Y.N. Takayabu and M. Murakami, The structure of super cloud clusters observed in 1-20 June 1986 and their relationship to easterly waves, J. Meteor. Soc. Japan, 69, 105-125, 1991.

[50] Y.N. Takayabu, Large-scale cloud disturbances associated with equatorial waves. Part I: spectral features of the cloud disturbances, J. Meteor. Soc. Japan, 72, 433-448, 1994.

[51] S.N. Tulich and B.E. Mapes, On the genesis and evolution of multi-scale convective wave patterns in a two- dimensional cloud-resolving model, J. Atmos. Sci., 65, 140-155, 2008.

[52] S.N. Tulich, D.A. Randall and B.E. Mapes, Vertical-mode and cloud decomposition of largescale convectively coupled gravity waves in a two-dimensional cloud-resolving model, J. Atmos. Sci., 64, 1210-1229, 2007.

[53] M. Wheeler and G.N. Kiladis, Convectively coupled equatorial waves: analysis of clouds and temperature in the wavenumber-frequency domain, J. Atmos. Sci., 56, 374-399, 1999.

[54] M. Wheeler, G.N. Kiladis and P.J. Webster, Large scale dynamical fields associated with convectively coupled equatorial waves, J. Atmos. Sci., 57, 613-640, 2000.

[55] E.J. Zipser, The role of organized unsaturated convective downdrafts in the structure and rapid decay of an equatorial disturbance, J. Appl. Meteor., 8, 799-814, 1969.

[56] E.J. Zipser, R.J. Meitin and M.A. LeMone, Mesoscale motion fields in association with a GATE convection band, J. Atmos. Sci., 38 , 1725-1750, 1981. 